\title{
36. BIOSTRATIGRAPHIC SYNTHESIS: LATE OLIGOCENE AND NEOGENE OF THE WESTERN TROPICAL PACIFIC
}

\author{
P. Bronnimann, University of Geneva, Geneva, Switzerland; \\ E. Martini, University of Frankfurt, Frankfurt, Germany; \\ J. Resig, Hawaii Institute of Geophysics, Honolulu, Hawaii; \\ W. R. Riedel and A. Sanfilippo, Scripps Institution of Oceanography, La Jolla, California; \\ and T. Worsley, University of Illinois, Urbana, Illinois
}

The drill-cores obtained by Leg 7 of the Deep-Sea Drilling expedition provide a basis for a detailed biostratigraphy of pelagic microfossil groups in the western tropical Pacific. Representation of the Neogene is practically complete (though siliceous microfossils are not satisfactorily preserved in the postMiocene sediments), and the Oligocene is represented by intermittent cores. Much work remains to be done on the microfossils of these drilled sequences, but results presented in preceding chapters on the planktonic foraminifera, calcareous nannofossils and radiolarians can be integrated to form a biostratigraphy of the late Oligocene and Neogene more detailed and comprehensive than that yet available for any other oceanic region.

The integrated results are here presented in two tables and a figure. Table 1 lists paleontological events (tops and bottoms of ranges of taxa) in stratigraphic order, as observed in the Leg 7 cores. This interrelated sequence of foraminiferal, calcareous nannofossil and radiolarian events offers the opportunity for very detailed correlation of western tropical Pacific sediments. Table 2 shows the relationships of the zonations used for the three principal microfossil groups investigated. Figure 1 presents a correlation of the sequences drilled at Sites 62 through 66 .

In Table 1, the lower limits of ranges of taxa are indicated as "B" (for bottoms) and the upper limits are indicated as " $\mathrm{T}$ " (for tops). Whether these limits are morphotypic or evolutionary can be determined by reference to the special chapters on the three microfossil groups. For the calcareous microfossil groups, the sequence of paleontological events presented in this table is that observed at Site 62 (for the upper part of the section) and at Site 64 (for the lower part of the section), and members of groups of events occurring at the same level (as far as can be determined from the information presently available for Sites 62 and 64) are arranged alphabetically according to the names of the taxa. For the radiolarians, the sequence of events presented is not the same as that observed at Sites 62 and 64 , if more reliable evidence on the sequence is available from other sites (see explanation of Table 6 in the chapter by Riedel and Sanfilippo). The level at which each paleontological event occurred is given in terms of core sections and (in parentheses) depth in meters below the sea floor at Site 62 or Site 64 .

Table 2 shows the relationship of the Neogene zonations based on calcareous nannofossils, pelagic foraminifera and radiolarians, as observed at Sites 62 , 63 and 64. The thicknesses of the zones as plotted are approximately proportional to the thicknesses at Sites 62 and 64 , and some idea of the durations can be gained from the Site Summary chapters, where they are plotted against a time scale. The columns on either side of the foraminiferal one are used to indicate (by crossed lines) ranges of uncertainty in the correlation of zonal boundaries, and hachured zones in the foraminiferal column indicate ranges of uncertainty in placement of " $\mathrm{N}$ " zonal boundaries.

Figure 1 presents a simplified correlation-diagram of the sequences at Sites 62 through 66 . The column for Site 65 is placed farthest to the right because its radiolarian assemblages are admixed by extensive reworking, and correlations are therefore subject to error. Only a few of the many possible correlation lines are included, in order that the diagram should remain legible. Solid lines are used to connect adjacent sites when a paleontological event occurred in cored intervals at both sites, and broken lines when an event occurred in an uncored interval at one or both of a pair of adjacent sites. Dotted lines across the site-column are used for certain radiolarian zonal boundaries in the lower parts of the sequences at Sites 64 and 65. Some correlation lines that cross are included to emphasize parts of the sequence requiring further investigation. 
TABLE 1

Sequence of Paleontological Events (From Youngest Downward)

Recognized in the Western Pacific Drill-Cores (For explanation, see text)

\begin{tabular}{|c|c|c|c|c|c|}
\hline \multicolumn{3}{|c|}{ Calcareous Nannofossil Events } & \multicolumn{2}{|c|}{ Foraminiferal Events } & Radiolarian Events \\
\hline \multirow[t]{15}{*}{ B } & $\begin{array}{l}\text { Emiliania } \\
\text { huxleyi }\end{array}$ & $\begin{array}{l}\text { Within } \\
62.1-1-1 \\
(7)\end{array}$ & & & \multirow{19}{*}{ 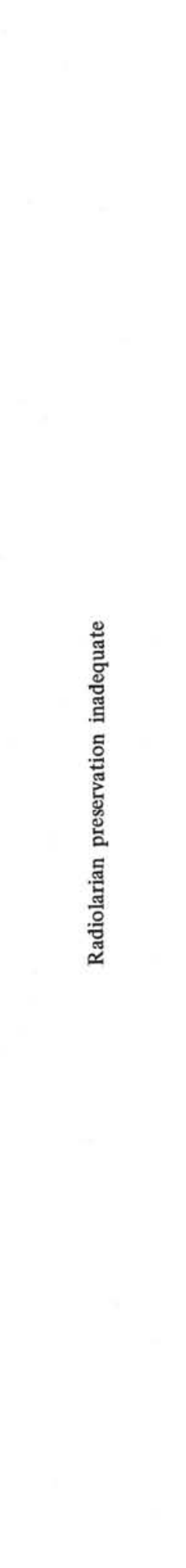 } \\
\hline & & & 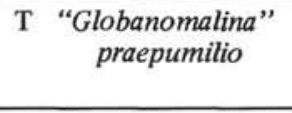 & $\begin{array}{c}62.1-1-1 \\
62.1-1-2 \\
(7-8) \\
\end{array}$ & \\
\hline & & & $\begin{array}{c}\text { T Globigerina } \\
\text { bulloides }\end{array}$ & $\begin{array}{c}62.1-1-1 \\
62.1-1-2 \\
(7-8) \\
\end{array}$ & \\
\hline & & & $\begin{array}{r}\text { T Globigerina } \\
\text { conglomerata } \\
\text { conglomerata }\end{array}$ & $\begin{array}{c}62.1-1-1 \\
62.1-1-2 \\
(7-8)\end{array}$ & \\
\hline & & & $\begin{array}{c}\text { T Globigerina } \\
\text { aff. falconensis } \\
\text { falconensis }\end{array}$ & $\begin{array}{c}62.1-1-1 \\
62.1-1-2 \\
(7-8)\end{array}$ & \\
\hline & & & $\begin{array}{c}\text { T Globigerinita } \\
\text { uvula group }\end{array}$ & $\begin{array}{c}62.1-1-1 \\
62.1-1-2 \\
(7-8) \\
\end{array}$ & \\
\hline & & & $\begin{array}{c}\mathrm{T} \text { Globigerinoides } \\
\text { quadrilobatus } \\
\text { immaturus }\end{array}$ & $\begin{array}{c}62.1-1-1 \\
62.1-1-2 \\
(7-8) \\
\end{array}$ & \\
\hline & & & $\begin{array}{c}\text { T Globorotalia } \\
\text { crassaformis } \\
\text { crassaformis }\end{array}$ & $\begin{array}{c}62.1-1-1 \\
62.1-1-2 \\
(7-8) \\
\end{array}$ & \\
\hline & & & $\begin{array}{c}\text { T Globorotalia } \\
\text { crassaformis } \\
\text { oceanica }\end{array}$ & $\begin{array}{c}62.1-1-1 \\
62.1-1-2 \\
(7-8)\end{array}$ & \\
\hline & & & $\begin{array}{c}\text { T } \begin{array}{c}\text { Globorotalia } \\
\text { pumilio }\end{array} \\
\end{array}$ & $\begin{array}{c}62.1-1-1 \\
62.1-1-2 \\
(7-8) \\
\end{array}$ & \\
\hline & & & $\begin{array}{l}\text { B Globorotalia } \\
\text { punticulata }\end{array}$ & $\begin{array}{c}62.1-1-1 \\
62.1-1-2 \\
(7-8) \\
\end{array}$ & \\
\hline & & & $\begin{array}{c}\text { B Hastigerina } \\
\text { adamsi }\end{array}$ & $\begin{array}{c}62.1-1-1 \\
62.1-1-2 \\
(7-8) \\
\end{array}$ & \\
\hline & & & $\begin{array}{c}\text { B Hastigerinella } \\
\text { digitata } \\
\text { digitata }\end{array}$ & $\begin{array}{c}62.1-1-1 \\
62.1-1-2 \\
(7-8) \\
\end{array}$ & \\
\hline & & & $\begin{array}{c}\text { T Candeina } \\
\text { nitida } \\
\text { praenitida }\end{array}$ & $\begin{array}{l}62.1-1-3 \\
62.1-2-1 \\
(10-16) \\
\end{array}$ & \\
\hline & & & $\begin{array}{r}\text { T Globigerina } \\
\text { decoraperta } \\
\text { decoraperta }\end{array}$ & $\begin{array}{l}62.1-1-3 \\
62.1-2-1 \\
(10-16)\end{array}$ & \\
\hline \multirow{4}{*}{\multicolumn{2}{|c|}{$\begin{array}{c}\text { Pseudoemiliana } \\
\text { lacunosa }\end{array}$}} & \multirow[t]{4}{*}{$\begin{array}{l}62.1-1-C C \\
62.1-2-1 \\
(15-16) \\
\end{array}$} & $\begin{array}{l}\mathrm{T} \text { Globigerina } \\
\text { aff. microstoma }\end{array}$ & $\begin{array}{l}62.1-1-3 \\
62.1-2-1 \\
(10-16)\end{array}$ & \\
\hline & & & $\begin{array}{l}\text { T Globigerina } \\
\text { pseudofoliata }\end{array}$ & $\begin{array}{c}62.1-1-3 \\
62.1-2-1 \\
(10-16) \\
\end{array}$ & \\
\hline & & & $\begin{array}{c}\text { T } \begin{array}{c}\text { Globigerinoides } \\
\text { elongatus }\end{array} \\
\text { to }\end{array}$ & $\begin{array}{l}62.1-1-3 \\
62.1-2-1 \\
(10-16)\end{array}$ & \\
\hline & & & $\begin{array}{r}\text { T Globorotalia } \\
\text { acostaensis } \\
\text { acostaensis }\end{array}$ & $\begin{array}{l}62.1-1-3 \\
62.1-2-1 \\
(10-16)\end{array}$ & \\
\hline
\end{tabular}


TABLE 1 - Continued

\begin{tabular}{|c|c|c|c|c|}
\hline \multicolumn{2}{|r|}{ Calcareous Nannofossil Events } & \multicolumn{2}{|c|}{ Foraminiferal Events } & Radiolarian Events \\
\hline & \multirow{15}{*}{$t$} & $\begin{array}{l}\mathrm{T} \text { Globorotalia } \\
\text { truncatulinoides }\end{array}$ & $\begin{array}{l}62.1-1-3 \\
62.1-2-1 \\
(10-16)\end{array}$ & \multirow{19}{*}{ 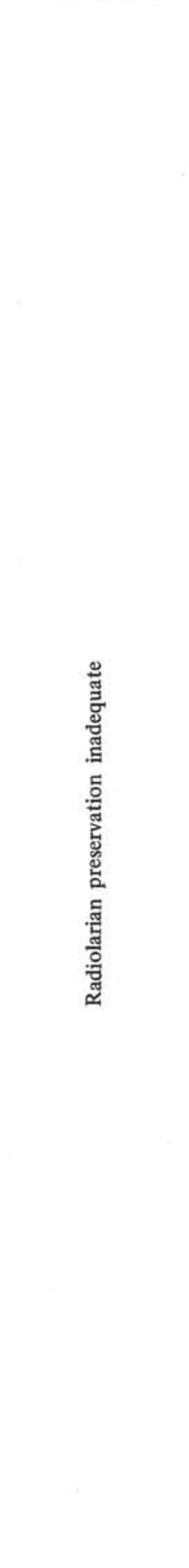 } \\
\hline & & $\begin{array}{c}\text { T Globorotalia } \\
\text { tumida } \\
\text { flexuosa }\end{array}$ & $\begin{array}{l}62.1-1-3 \\
62.1-2-1 \\
(10-16)\end{array}$ & \\
\hline & & $\begin{array}{c}\mathrm{T} \text { Globorotalia } \\
\text { tumida } \\
\text { lata }\end{array}$ & $\begin{array}{l}62.1-1-3 \\
62.1-2-1 \\
(10-16)\end{array}$ & \\
\hline & & $\begin{array}{c}\mathrm{T} \text { Globigerinoides } \\
\text { bollii }\end{array}$ & $\begin{array}{l}62.1-2-1 \\
62.1-2-2 \\
(16-17) \\
\end{array}$ & \\
\hline & & $\begin{array}{c}\mathrm{T} \text { Orbulina } \\
\text { suturalis }\end{array}$ & $\begin{array}{l}62.1-2-1 \\
62.1-2-2 \\
(16-17)\end{array}$ & \\
\hline & & $\begin{array}{c}\text { T Globigerinoides } \\
\text { quadrilobatus } \\
\text { trilobus }\end{array}$ & $\begin{array}{l}62.1-2-3 \\
62.1-2-4 \\
(19-20)\end{array}$ & \\
\hline & & $\begin{array}{c}\text { T Globorotalia } \\
\text { tosaensis }\end{array}$ & $\begin{array}{l}62.1-2-4 \\
62.1-3-2 \\
(20-30)\end{array}$ & \\
\hline & & $\begin{array}{c}\text { B } \begin{array}{c}\text { Globorotalia } \\
\text { hirsuta }\end{array} \\
\text {. }\end{array}$ & $\begin{array}{l}62.1-3-2 \\
62.1-4-1 \\
(30-35)\end{array}$ & \\
\hline & & $\begin{array}{c}\text { T Globorotalia } \\
\text { inflata }\end{array}$ & $\begin{array}{l}62.1-3-2 \\
62.1-4-1 \\
(30-35)\end{array}$ & \\
\hline & & $\begin{array}{r}\text { T Globorotalia } \\
\text { planispira }\end{array}$ & $\begin{array}{l}62.1-3-2 \\
62.1-4-1 \\
(30-35)\end{array}$ & \\
\hline & & $\begin{array}{l}\text { T Globorotalia } \\
\text { pseudopumilio }\end{array}$ & $\begin{array}{l}62.1-3-2 \\
62.1-4-1 \\
(30-35)\end{array}$ & \\
\hline & & $\begin{array}{l}\text { T Globigerina } \\
\text { aff. quinqueloba }\end{array}$ & $\begin{array}{l}62.1-4-1 \\
62.1-4-2 \\
(35-36)\end{array}$ & \\
\hline & & $\begin{array}{c}\mathrm{T} \text { Sphaeroidinellopsis } \\
\text { seminulina } \\
\text { seminulina }\end{array}$ & $\begin{array}{l}62.1-4-1 \\
62.1-4-2 \\
(35-36)\end{array}$ & \\
\hline & & $\begin{array}{l}\text { T Globigerina } \\
\text { falconensis } \\
\text { falconensis }\end{array}$ & $\begin{array}{l}62.1-4-5 \\
62.1-4-6 \\
(41-42)\end{array}$ & \\
\hline & & $\begin{array}{c}\text { T Globigerina } \\
\text { falconensis } \\
\text { palpebra }\end{array}$ & $\begin{array}{l}62.1-4-5 \\
62.1-4-6 \\
(41-42)\end{array}$ & \\
\hline $\bar{T}$ & $\begin{array}{l}\text { Discoaster } \\
\quad \text { brouweri }\end{array}$ & $\begin{array}{l}\text { T Globigerinoides } \\
\text { quadrilobatus } \\
\text { fistulosus }\end{array}$ & $\begin{array}{l}62.1-4-5 \\
62.1-4-6 \\
(41-42)\end{array}$ & \\
\hline \multirow{3}{*}{\multicolumn{2}{|c|}{$\begin{array}{c}\text { B Gephyrocapsa } \\
\text { oceanica }\end{array}$}} & & & \\
\hline & & $\begin{array}{l}\text { B Globorotalia } \\
\text { truncatulinoides (?) }\end{array}$ & $\begin{array}{l}62.1-5-1 \\
62.1-5-2 \\
(44-45)\end{array}$ & \\
\hline & & $\begin{array}{c}\text { B Pulleniatina } \\
\text { obliquiloculata } \\
\text { finalis }\end{array}$ & $\begin{array}{l}62.1-5-1 \\
62.1-5-2 \\
(44-45)\end{array}$ & \\
\hline
\end{tabular}


TABLE 1 - Continued

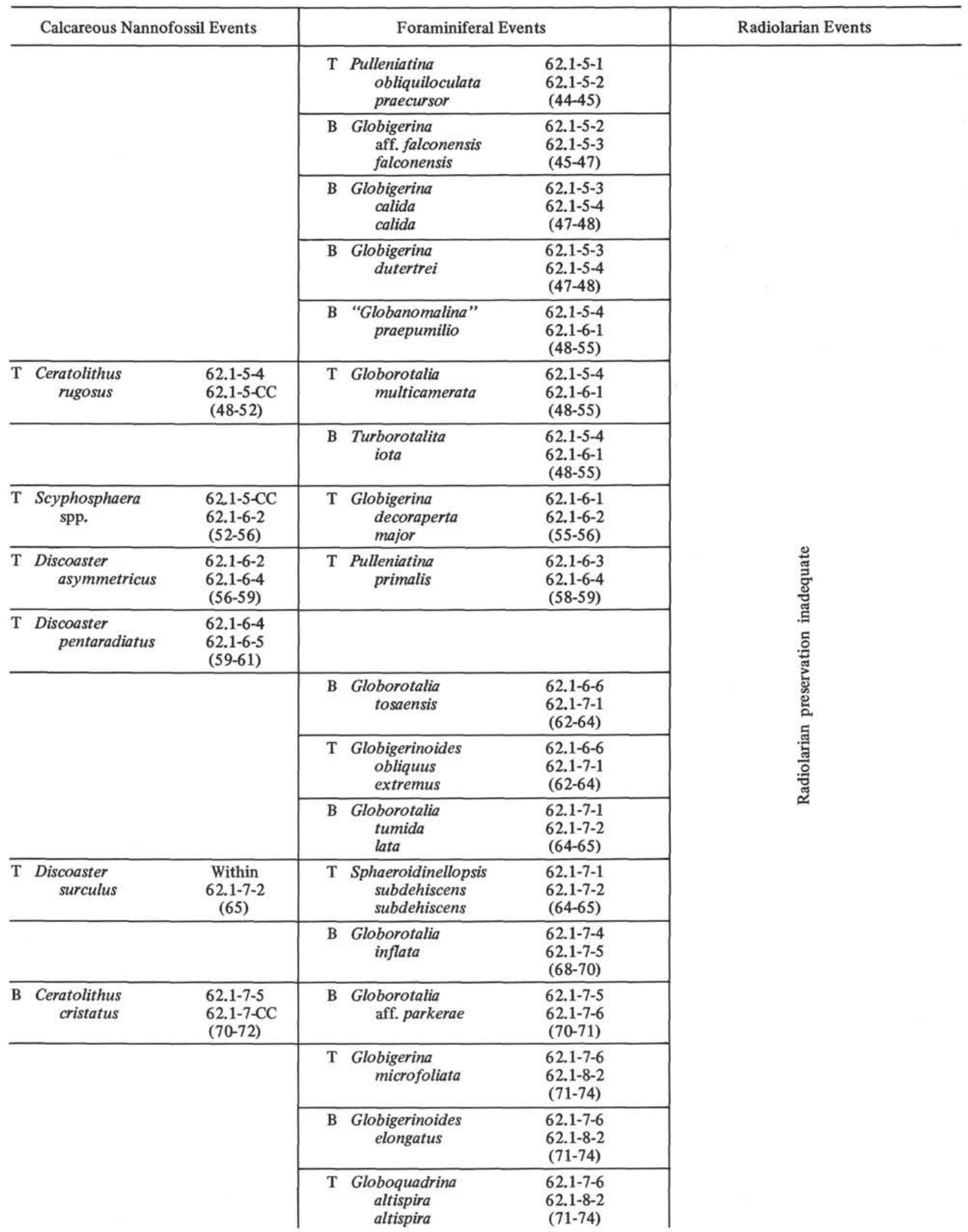


TABLE 1 - Continued

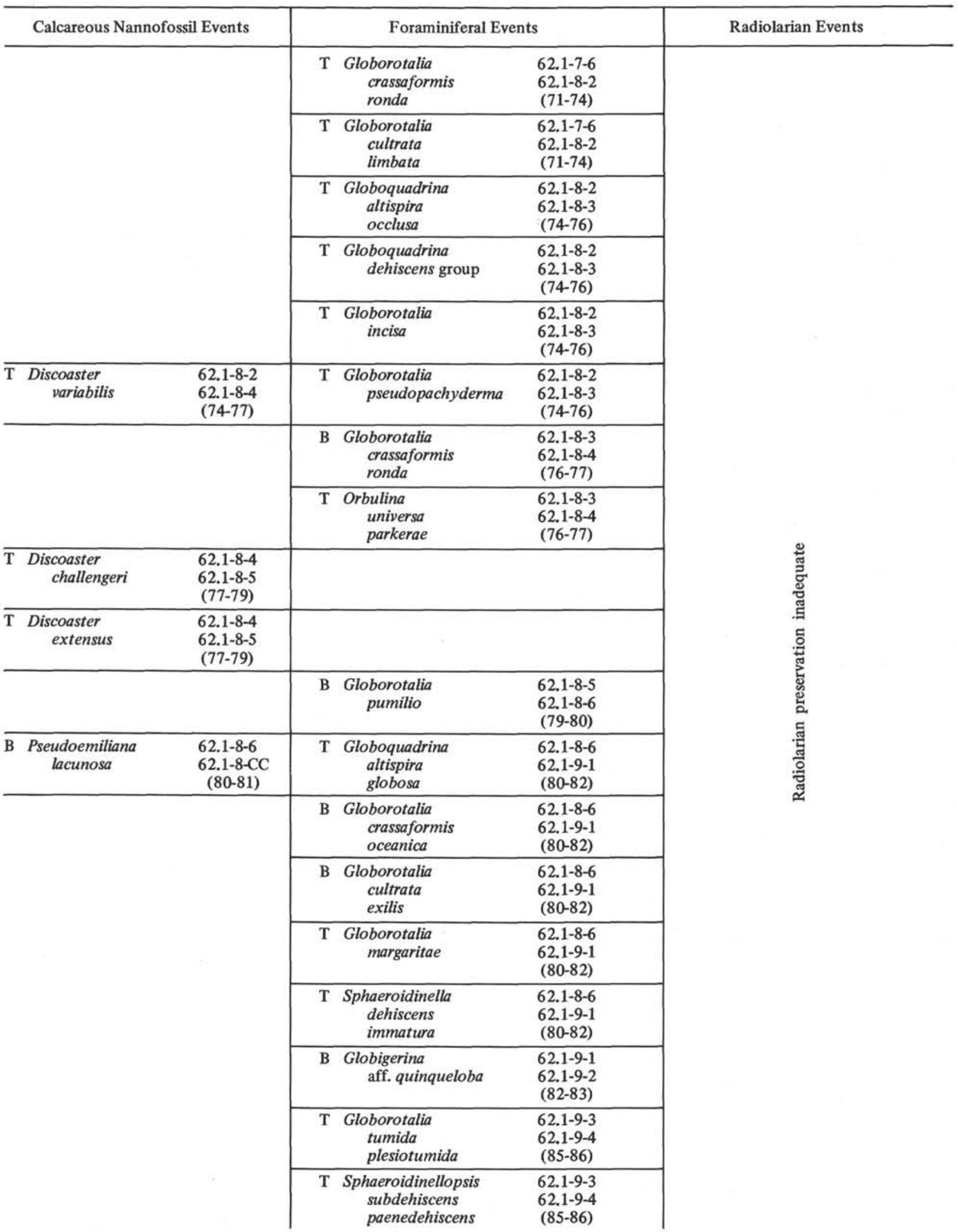


TABLE 1 - Continued

\begin{tabular}{|c|c|c|c|c|c|}
\hline \multicolumn{3}{|c|}{ Calcareous Nannofossil Events } & \multicolumn{2}{|c|}{ Foraminiferal Events } & Radiolarian Events \\
\hline $\mathrm{T}$ & $\begin{array}{l}\text { Reticulofenestra } \\
\quad \text { pseudoumbilica }\end{array}$ & $\begin{array}{l}62.1-9-6 \\
62.1-9-\mathrm{CC} \\
(86-90)\end{array}$ & $\begin{array}{c}\text { B Globigerinoides } \\
\text { ruber }\end{array}$ & $\begin{array}{l}62.1-9-4 \\
62.1-10-1 \\
(86-93)\end{array}$ & \multirow{19}{*}{ 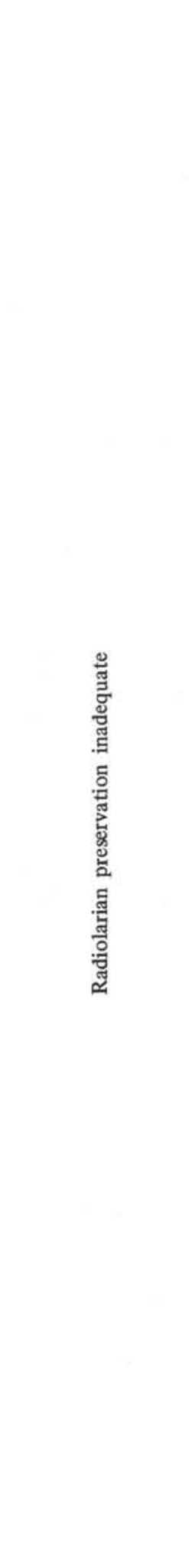 } \\
\hline \multirow[t]{9}{*}{ B } & $\begin{array}{l}\text { Scyphosphaera } \\
\text { spp. }\end{array}$ & $\begin{array}{c}62.1-9-\mathrm{CC} \\
62.1-10-1 \\
(90-93)\end{array}$ & $\begin{array}{r}\text { B Globorotalia } \\
\text { crassaformis } \\
\text { crassaformis }\end{array}$ & $\begin{array}{l}62.1-9-4 \\
62.1-10-1 \\
(86-93)\end{array}$ & \\
\hline & & & 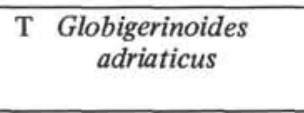 & $\begin{array}{l}62.1-10-1 \\
62.1-10-2 \\
(93-94) \\
\end{array}$ & \\
\hline & & & $\begin{array}{c}\text { B Sphaeroidinella } \\
\text { dehiscens } \\
\text { dehiscens }\end{array}$ & $\begin{array}{l}62.1-10-2 \\
62.1-10-3 \\
(94-96)\end{array}$ & \\
\hline & & & $\begin{array}{c}\text { T Globorotalia } \\
\text { hirsuta } \\
\text { praehirsuta }\end{array}$ & $\begin{array}{l}62.1-10-3 \\
62.1-10-4 \\
(96-97) \\
\end{array}$ & \\
\hline & & & $\begin{array}{r}\text { B Globorotalia } \\
\text { planispira }\end{array}$ & $\begin{array}{l}62.1-10-3 \\
62.1-10-4 \\
(96-97) \\
\end{array}$ & \\
\hline & & & $\begin{array}{l}\text { B Globorotalia } \\
\text { pseudopumilio }\end{array}$ & $\begin{array}{l}62.1-10-5 \\
62.1-10-6 \\
(99-100) \\
\end{array}$ & \\
\hline & & & $\begin{array}{r}\text { B Pulleniatina } \\
\text { obliquiloculata } \\
\text { obliquiloculata }\end{array}$ & $\begin{array}{l}62.1-10-5 \\
62.1-10-6 \\
(99-100)\end{array}$ & \\
\hline & & & $\begin{array}{r}\mathrm{T} \text { Pulleniatina } \\
\text { praepulleniatina }\end{array}$ & $\begin{array}{r}62.1-10-5 \\
62.1-10-6 \\
(99-100) \\
\end{array}$ & \\
\hline & & & $\begin{array}{c}\text { B Pulleniatina } \\
\text { obliquiloculata } \\
\text { praecursor }\end{array}$ & $\begin{array}{l}62.1-10-6 \\
62.1-11-1 \\
(100-102)\end{array}$ & \\
\hline \multirow[t]{9}{*}{ B } & \multirow[t]{9}{*}{$\begin{array}{l}\text { Discoaster } \\
\quad \text { asymmetricus }\end{array}$} & \multirow[t]{9}{*}{$\begin{array}{l}62.1-11-1 \\
62.1-11-2 \\
(102-103) \\
\end{array}$} & $\begin{array}{c}\mathrm{T} \text { Globigerina } \\
\text { conglomerata } \\
\text { venezuelana }\end{array}$ & $\begin{array}{l}62.1-11-1 \\
62.1-11-2 \\
(102-103) \\
\end{array}$ & \\
\hline & & & $\begin{array}{r}\text { T Orbulina } \\
\text { universa } \\
\text { bilobata }\end{array}$ & $\begin{array}{l}62.1-11-1 \\
62.1-11-2 \\
(102-103) \\
\end{array}$ & \\
\hline & & & $\begin{array}{c}\text { T Sphaerodinellopsis } \\
\text { seminulina } \\
\text { kochi }\end{array}$ & $\begin{array}{l}62.1-11-1 \\
62.1-11-2 \\
(102-103)\end{array}$ & \\
\hline & & & $\begin{array}{l}\text { T Pulleniatina } \\
\text { spectabilis } \\
\text { praespectabilis }\end{array}$ & $\begin{array}{l}62.1-11-3 \\
62.1-11-4 \\
(105-106)\end{array}$ & \\
\hline & & & $\begin{array}{r}\text { T Pulleniatina } \\
\text { spectabilis } \\
\text { spectabilis }\end{array}$ & $\begin{array}{l}62.1-11-3 \\
62.1-11-4 \\
(105-106) \\
\end{array}$ & \\
\hline & & & $\begin{array}{c}\text { T Globigerina } \\
\text { nepenthes } \\
\text { nepenthes }\end{array}$ & $\begin{array}{l}62.1-11-4 \\
62.1-11-5 \\
(106-108)\end{array}$ & \\
\hline & & & $\begin{array}{l}\text { B Globigerina } \\
\text { pseudofoliata }\end{array}$ & $\begin{array}{l}62.1-11-5 \\
62.1-11-6 \\
(108-109) \\
\end{array}$ & \\
\hline & & & $\begin{array}{c}\mathrm{T} \text { Globorotalia } \\
\text { acostaensis } \\
\text { tegillata }\end{array}$ & $\begin{array}{l}62.1-11-6 \\
62.1-12-1 \\
(109-111)\end{array}$ & \\
\hline & & & $\begin{array}{r}\text { B Pulleniatina } \\
\text { spectabilis } \\
\text { spectabilis }\end{array}$ & $\begin{array}{l}62.1-11-6 \\
62.1-12-1 \\
(109-111)\end{array}$ & \\
\hline
\end{tabular}


TABLE 1 - Continued

\begin{tabular}{|c|c|c|c|c|c|}
\hline \multicolumn{2}{|r|}{ Calcareous Nannofossil Events } & \multicolumn{2}{|c|}{ Foraminiferal Events } & \multicolumn{2}{|c|}{ Radiolarian Events } \\
\hline \multirow{10}{*}{\multicolumn{2}{|c|}{ s. }} & $\begin{array}{l}\text { T Globorotalia } \\
\text { merotumida }\end{array}$ & $\begin{array}{l}62.1-12-1 \\
62.1-12-2 \\
(111-112)\end{array}$ & & \\
\hline & & $\begin{array}{r}\text { B Globigerinoides } \\
\text { quadrilobatus } \\
\text { sacculifer } \quad \alpha\end{array}$ & $\begin{array}{l}62.1-12-2 \\
62.1-12-3 \\
(112-114)\end{array}$ & & \\
\hline & & $\begin{array}{c}\text { T Hastigerina } \\
\text { siphonifera } \\
\text { involuta }\end{array}$ & $\begin{array}{l}62.1-12-2 \\
62.1-12-3 \\
(112-114)\end{array}$ & & \\
\hline & & $\begin{array}{c}\text { B Globorotalia } \\
\text { ungulata }\end{array}$ & $\begin{array}{l}62.1-12-3 \\
62.1-12-4 \\
(114-115) \\
\end{array}$ & 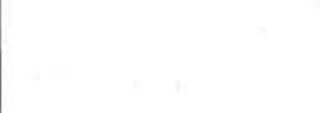 & \\
\hline & & $\begin{array}{r}\text { T Globigerina } \\
\text { nepenthes } \\
\text { picassiana }\end{array}$ & $\begin{array}{l}62.1-12-4 \\
62.1-12-5 \\
(115-117)\end{array}$ & & \\
\hline & & $\begin{array}{l}\text { B Globigerina } \\
\text { microfoliata }\end{array}$ & $\begin{array}{l}62.1-12-5 \\
62.1-12-6 \\
(117-118)\end{array}$ & 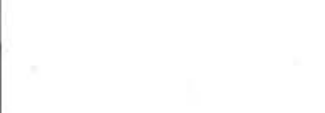 & \\
\hline & & $\begin{array}{l}\text { B Globigerina } \\
\text { rubescens }\end{array}$ & $\begin{array}{l}62.1-12-6 \\
62.1-13-1 \\
(118-120)\end{array}$ & & \\
\hline & & $\begin{array}{c}\text { B Sphaeroidinella } \\
\text { dehiscens } \\
\text { immatura }\end{array}$ & $\begin{array}{l}62.1-12-6 \\
62.1-13-1 \\
(118-120)\end{array}$ & & \\
\hline & & $\begin{array}{r}\text { T Globigerina } \\
\text { nepenthes } \\
\text { delicatula }\end{array}$ & $\begin{array}{l}62.1-13-1 \\
62.1-13-2 \\
(120-121)\end{array}$ & & \\
\hline & & $\begin{array}{r}\text { B Globorotalia } \\
\text { margaritae }\end{array}$ & $\begin{array}{l}62.1-13-3 \\
62.1-13-4 \\
(123-124)\end{array}$ & $\begin{array}{l}\text { Spongaster klingi } \\
\text { S. pentas }\end{array}$ & $\begin{array}{l}62.1-14-\mathrm{S} \\
62.1-18-\mathrm{CC} \\
(136-174) \\
\end{array}$ \\
\hline \multirow[t]{8}{*}{ B } & $\begin{array}{l}62.1-13-4 \\
62.1-13-5 \\
(124-126)\end{array}$ & $\begin{array}{l}\text { B Globorotalia } \\
\text { pseudopachyderma }\end{array}$ & $\begin{array}{l}62.1-13-4 \\
62.1-13-5 \\
(124-126)\end{array}$ & $\begin{array}{l}\text { Ommatartus penultimus } \\
\text { O. avitus }\end{array}$ & $\begin{array}{l}62.1-13-3 \\
62.1-13-5 \\
(123-126)\end{array}$ \\
\hline & & $\begin{array}{c}\text { B Globorotalia } \\
\text { incisa }\end{array}$ & $\begin{array}{l}62.1-14-1 \\
62.1-14-2 \\
(130-131) \\
\end{array}$ & & \\
\hline & & $\begin{array}{r}\text { B Globorotalia } \\
\text { pseudopima }\end{array}$ & $\begin{array}{l}62.1-14-4 \\
62.1-14-5 \\
(134-136) \\
\end{array}$ & & \\
\hline & & $\begin{array}{r}\text { T Globorotalia } \\
\text { cibaoensis }\end{array}$ & $\begin{array}{l}62.1-15-1 \\
62.1-15-2 \\
(139-140)\end{array}$ & & \\
\hline & & $\begin{array}{l}\text { B Globorotalia } \\
\text { aff. subscitula }\end{array}$ & $\begin{array}{l}62.1-15-1 \\
62.1-15-2 \\
(139-140)\end{array}$ & & \\
\hline & & $\begin{array}{r}\text { B Globigerina } \\
\text { conglomerata } \\
\text { conglomerata }\end{array}$ & $\begin{array}{l}62.1-15-2 \\
62.1-15-3 \\
(140-142) \\
\end{array}$ & & \\
\hline & & $\begin{array}{c}\text { B Globorotalia } \\
\text { tumida } \\
\text { flexuosa }\end{array}$ & $\begin{array}{l}62.1-15-4 \\
62.1-15-5 \\
(143-145)\end{array}$ & & \\
\hline & & $\begin{array}{c}\text { B Globorotalia } \\
\text { tumida } \\
\text { tumida }\end{array}$ & $\begin{array}{l}62.1-15-4 \\
62.1-15-5 \\
(143-145)\end{array}$ & & \\
\hline $\bar{T}$ & $\begin{array}{l}\text { Discoaster } \\
\quad \text { quinqueramus }\end{array}$ & $\begin{array}{c}\text { B Globogerina } \\
\text { falconensis } \\
\text { palpebra }\end{array}$ & $\begin{array}{l}62.1-15-5 \\
62.1-15-6 \\
(145-146)\end{array}$ & & \\
\hline
\end{tabular}


TABLE 1 - Continued

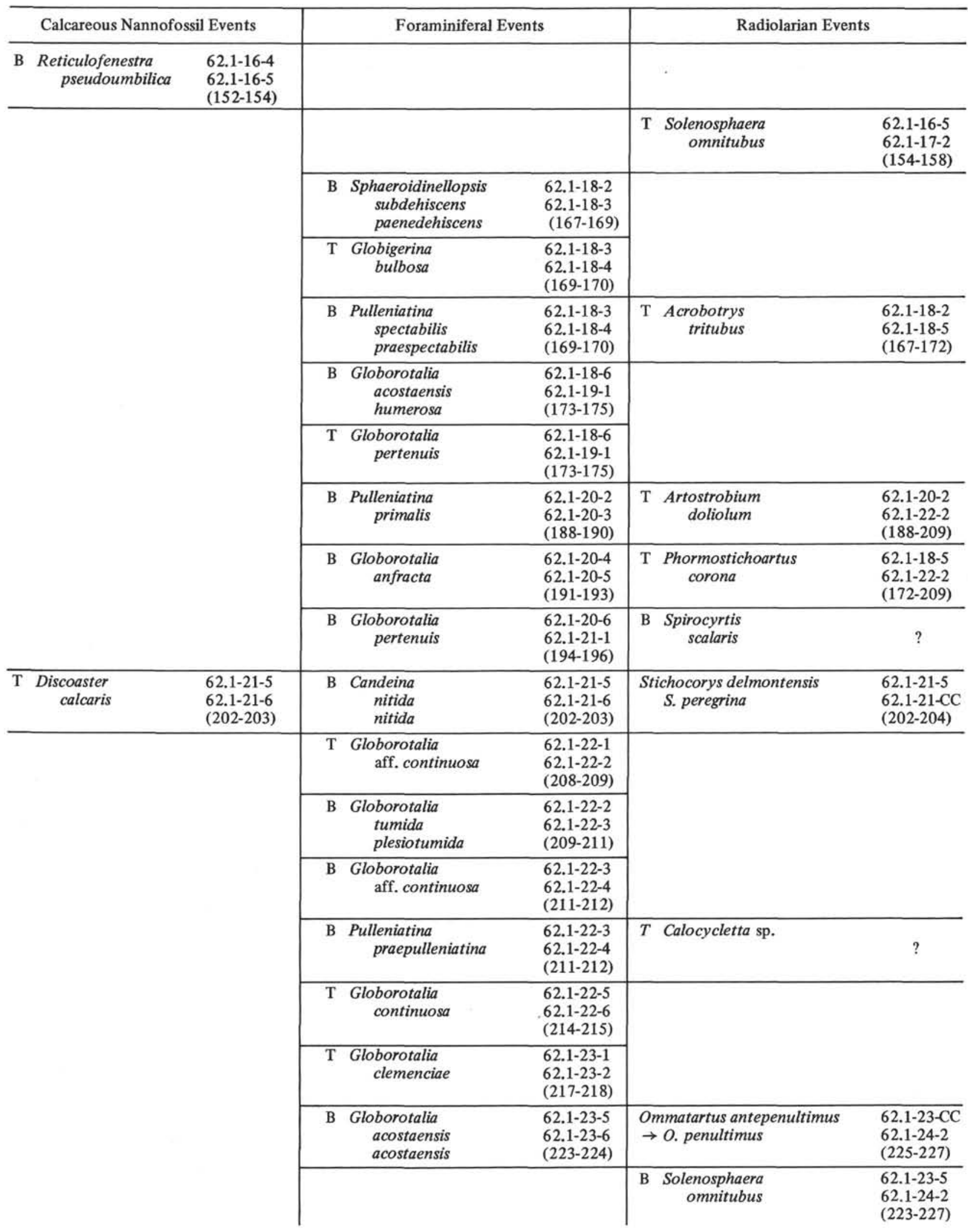


TABLE 1 - Continued

\begin{tabular}{|c|c|c|c|c|c|}
\hline \multicolumn{2}{|c|}{ Calcareous Nannofossil Events } & \multicolumn{2}{|c|}{ Foraminiferal Events } & \multicolumn{2}{|l|}{ Radiolarian Events } \\
\hline & & $\begin{array}{r}\text { B Globigerinoides } \\
\text { conglobatus }\end{array}$ & $\begin{array}{l}62.1-24-4 \\
62.1-24-5 \\
(230-232)\end{array}$ & & \\
\hline & & $\begin{array}{r}\text { B Globorotalia } \\
\text { cibaoensis }\end{array}$ & $\begin{array}{l}62.1-24-6 \\
62.1-25-1 \\
(233-235)\end{array}$ & $\begin{array}{c}\mathrm{T} \text { Ommatartus } \\
\text { hughesi }\end{array}$ & $\begin{array}{l}62.1-24-5 \\
62.1-24-C C \\
(232-234)\end{array}$ \\
\hline & & $\begin{array}{l}\mathrm{T} \text { Globorotalia } \\
\text { aff. apertura }\end{array}$ & $\begin{array}{l}62.1-25-1 \\
62.1-25-2 \\
(235-236)\end{array}$ & & \\
\hline $\begin{array}{l}\mathrm{T} \text { Triquetrorhabdulus } \\
\text { rugosus }\end{array}$ & $\begin{array}{l}\text { Within } \\
62.1-25-5 \\
(241)\end{array}$ & & & $\begin{array}{l}\text { Cyclampterium brachythorax } \\
\rightarrow \text { C. neatum }\end{array}$ & $\begin{array}{l}62.1-25-2 \\
62.1-26-2 \\
(236-247) \\
\end{array}$ \\
\hline & & $\begin{array}{l}\text { B Globorotalia } \\
\text { hirsuta } \\
\text { praehirsuta }\end{array}$ & $\begin{array}{l}62.1-27-1 \\
62.1-27-2 \\
(255-256)\end{array}$ & & \\
\hline & & & & $\begin{array}{r}\text { B Acrobotrys } \\
\text { tritubus }\end{array}$ & $\begin{array}{l}62.1-27-2 \\
62.1-27-4 \\
(256-259) \\
\end{array}$ \\
\hline \multirow[t]{2}{*}{ B $D$} & $\begin{array}{l}62.1-27-5 \\
62.1-28-2 \\
(261-265) \\
\end{array}$ & & & $\begin{array}{r}\mathrm{T} \text { Dictyocoryne } \\
\text { ontogensis }\end{array}$ & $\begin{array}{l}62.1-27-4 \\
62.1-28-2 \\
(259-265) \\
\end{array}$ \\
\hline & & $\begin{array}{l}\text { T Globigerina } \\
\text { aff. globorotaloidea }\end{array}$ & $\begin{array}{l}62.1-28-2 \\
62.1-28-3 \\
(265-267) \\
\end{array}$ & & \\
\hline $\begin{array}{l}\mathrm{T} \text { Discoaster } \\
\quad \text { adamanteus }\end{array}$ & $\begin{array}{l}62.1-28-3 \\
62.1-28-4 \\
(267-268)\end{array}$ & & & & \\
\hline $\begin{array}{l}\text { B Discoaster } \\
\text { surculus }\end{array}$ & $\begin{array}{l}\text { Within } \\
62.1-28-5 \\
(270)\end{array}$ & & & & \\
\hline \multirow[t]{9}{*}{$\begin{array}{r}\mathrm{T} \text { Coccolithus } \\
\text { tenuistriatus }\end{array}$} & $\begin{array}{l}62.1-29-2 \\
62.1-29-3 \\
(273-275) \\
\end{array}$ & & & B Spongaster & $\begin{array}{l}62.1-28-5 \\
62.1-29-5 \\
(270-278) \\
\end{array}$ \\
\hline & & $\begin{array}{c}\mathrm{T} \text { Globoquadrina } \\
\text { altispira } \\
\text { comica }\end{array}$ & $\begin{array}{l}62.1-29-3 \\
62.1-29-4 \\
(275-276)\end{array}$ & & \\
\hline & & $\begin{array}{c}\text { B Globigerina } \\
\text { calida } \\
\text { praecalida }\end{array}$ & $\begin{array}{l}62.1-29-4 \\
62.1-29-5 \\
(276-278) \\
\end{array}$ & & \\
\hline & & $\begin{array}{c}\text { B Orbulina } \\
\text { universa } \\
\text { parkerae }\end{array}$ & $\begin{array}{l}62.1-29-4 \\
62.1-29-5 \\
(276-278)\end{array}$ & & \\
\hline & & $\begin{array}{c}\text { B Globorotalia } \\
\text { acostaensis } \\
\text { tegillata }\end{array}$ & $\begin{array}{l}62.1-29-5 \\
62.1-29-6 \\
(278-279) \\
\end{array}$ & & \\
\hline & & $\begin{array}{c}\mathrm{T} \text { Globorotalia } \\
\text { lenguaensis }\end{array}$ & $\begin{array}{l}62.1-29-5 \\
62.1-29-6 \\
(278-279)\end{array}$ & & \\
\hline & & $\begin{array}{l}\text { B Candeina } \\
\text { nitida } \\
\text { praenitida }\end{array}$ & $\begin{array}{l}62.1-30-1 \\
62.1-30-2 \\
(282-283)\end{array}$ & $\begin{array}{l}\text { Cannartus petterssoni } \\
\rightarrow \text { Ommatartus hughesi }\end{array}$ & $\begin{array}{l}62.1-29-C C \\
62.1-30-2 \\
(280-283)\end{array}$ \\
\hline & & $\begin{array}{c}\text { B Globigerina } \\
\text { nepenthes } \\
\text { delicatula }\end{array}$ & $\begin{array}{l}62.1-30-3 \\
62.1-30-4 \\
(285-286)\end{array}$ & $\begin{array}{c}\text { T Liriospyris } \\
\text { elevata }\end{array}$ & $\begin{array}{l}62.1-30-2 \\
62.1-30-5 \\
(283-288)\end{array}$ \\
\hline & & $\begin{array}{r}\text { B } \begin{array}{c}\text { Globigerina } \\
\text { bulloides }\end{array} \\
\end{array}$ & $\begin{array}{l}62.1-30-4 \\
62.1-30-5 \\
(286-288)\end{array}$ & $\begin{array}{l}\text { B Carpocanarium } \\
\text { spp. }\end{array}$ & $\begin{array}{l}62.1-32-2 \\
62.0-3-6 \\
(303-307)\end{array}$ \\
\hline
\end{tabular}


TABLE 1 - Continued

\begin{tabular}{|c|c|c|c|c|c|c|}
\hline \multicolumn{3}{|c|}{ Calcareous Nannofossil Events } & \multicolumn{2}{|c|}{ Foraminiferal Events } & \multicolumn{2}{|c|}{ Radiolarian Events } \\
\hline $\mathrm{T}$ & $\begin{array}{l}\text { Discoaster } \\
\text { bollii }\end{array}$ & $\begin{array}{l}62.1-30-5 \\
62.1-30-C \mathrm{C} \\
(288-290)\end{array}$ & & & & \\
\hline $\mathrm{T}$ & $\begin{array}{l}\text { Discoaster } \\
\quad \text { hamatus }\end{array}$ & $\begin{array}{l}62.1-30-\mathrm{CC} \\
62.1-31-1 \\
(290-292)\end{array}$ & $\begin{array}{l}\text { B Globigerina } \\
\text { aff. } \\
\text { globorotaloidea }\end{array}$ & $\begin{array}{l}62.1-30-6 \\
62.1-31-1 \\
(289-292)\end{array}$ & $\begin{array}{c}\text { B Dictyocoryne } \\
\text { ontongensis }\end{array}$ & $\begin{array}{l}62.1-30-5 \\
62.1-31-2 \\
(288-293)\end{array}$ \\
\hline$T$ & $\begin{array}{l}\text { Catinaster } \\
\text { calyculus }\end{array}$ & $\begin{array}{l}62.1-31-2 \\
62.1-31-3 \\
(293-295) \\
\end{array}$ & & & & \\
\hline & \multirow[b]{4}{*}{. } & & $\begin{array}{l}\text { B Globorotalia } \\
\\
\end{array}$ & $\begin{array}{l}62.1-31-3 \\
62.1-31-4 \\
(295-296)\end{array}$ & $\begin{array}{l}\text { Cannartus laticonus } \\
\rightarrow \text { Ommatortus } \\
\text { antepenultimus }\end{array}$ & $\begin{array}{l}62.1-30-\mathrm{CC} \\
62.1-31-5 \\
(290-298)\end{array}$ \\
\hline & & & $\begin{array}{l}\text { B Globorotalia } \\
\text { aff. apertura }\end{array}$ & $\begin{array}{l}62.1-31-3 \\
62.1-31-4 \\
(295-296)\end{array}$ & & \\
\hline & & & $\begin{array}{c}\text { B Hastigerina } \\
\text { siphonifera } \\
\text { involuta }\end{array}$ & $\begin{array}{l}62.1-31-4 \\
62.1-31-5 \\
(296-298)\end{array}$ & & \\
\hline & & & $\begin{array}{c}\mathrm{T} \text { Globorotalia } \\
\text { siakensis }\end{array}$ & $\begin{array}{l}62.1-31-6 \\
62.1-32-1 \\
(299-302)\end{array}$ & & \\
\hline $\mathrm{T}$ & $\begin{array}{r}\text { Discoaster } \\
\text { aulakos }\end{array}$ & $\begin{array}{l}62.1-32-1 \\
62.1-32-2 \\
(302-303)\end{array}$ & $\begin{array}{c}\text { B Globigerinoides } \\
\text { obliquus } \\
\text { extremus }\end{array}$ & $\begin{array}{l}62.1-32-1 \\
62.1-32-2 \\
(302-303)\end{array}$ & & \\
\hline \multicolumn{2}{|c|}{$\begin{array}{r}\text { Catinaster } \\
\text { coalitus }\end{array}$} & $\begin{array}{l}62.1-32-3 \\
62.1-32-4 \\
(305-306)\end{array}$ & & & & \\
\hline $\bar{B}$ & $\begin{array}{l}\text { Discoaster } \\
\text { pentaradiatus }\end{array}$ & $\begin{array}{l}\text { Within } \\
62.1-32-5 \\
(308)\end{array}$ & $\begin{array}{c}\text { B Globoquadrina } \\
\text { altispira } \\
\text { comica }\end{array}$ & $\begin{array}{l}62.1-32-4 \\
62.1-32-5 \\
(306-308)\end{array}$ & & \\
\hline $\mathrm{T}$ & $\begin{array}{l}\text { Discoaster } \\
\quad \text { pseudovariabilis }\end{array}$ & $\begin{array}{l}\text { Within } \\
62.1-32-5 \\
(308)\end{array}$ & & & & \\
\hline \multirow[t]{5}{*}{$\bar{B}$} & \multirow[t]{5}{*}{$\begin{array}{c}\text { Discoaster } \\
\text { bollii }\end{array}$} & \multirow[t]{5}{*}{$\begin{array}{l}62.1-32-5 \\
62.1-32-6 \\
(308-309)\end{array}$} & $\begin{array}{r}\text { B Globigerina } \\
\text { decoraperta } \\
\text { decoraperta }\end{array}$ & $\begin{array}{l}62.1-32-5 \\
62.1-32-6 \\
(308-309)\end{array}$ & & \\
\hline & & & $\begin{array}{c}\text { B } \begin{array}{c}\text { Globigerina } \\
\text { decoraperta } \\
\text { major }\end{array} \\
\end{array}$ & $\begin{array}{l}62.1-32-5 \\
62.1-32-6 \\
(308-309)\end{array}$ & $\begin{array}{l}\text { Lithopera neotera } \\
\text { L. bacca }\end{array}$ & $\begin{array}{l}62.1-32-2 \\
62.1-34-3 \\
(303-324)\end{array}$ \\
\hline & & & $\begin{array}{r}\text { B Globigerina } \\
\text { nepenthes } \\
\text { nepenthes }\end{array}$ & $\begin{array}{l}62.1-32-5 \\
62.1-32-6 \\
(308-309) \\
\end{array}$ & $\begin{array}{c}\text { T Carpocanopsis } \\
\text { cristatum }\end{array}$ & $\begin{array}{l}62.1-31-5 \\
62.1-33-2 \\
(298-312) \\
\end{array}$ \\
\hline & & & $\mathrm{T} \begin{array}{c}\text { Globorotalia } \\
\text { riedeli }\end{array}$ & $\begin{array}{l}62.1-32-5 \\
62.1-32-6 \\
(308-309) \\
\end{array}$ & $\begin{array}{l}\text { T Lithopera } \\
\text { thornburgi }\end{array}$ & $\begin{array}{l}62.1-35-2 \\
62.1-35-5 \\
(329-334) \\
\end{array}$ \\
\hline & & & $\begin{array}{c}\mathrm{T} \text { Globigerina } \\
\text { druryi }\end{array}$ & $\begin{array}{l}62.1-32-6 \\
62.1-33-2 \\
(309-312)\end{array}$ & $\begin{array}{r}\text { T Lithopera } \\
\text { baueri }\end{array}$ & $\begin{array}{l}62.1-32-5 \\
62.1-33-2 \\
(308-312)\end{array}$ \\
\hline \multicolumn{2}{|c|}{$\begin{array}{r}\text { Tiscoaster } \\
\text { kugleri }\end{array}$} & \multicolumn{3}{|l|}{$\begin{array}{l}62.1-33-2 \\
62.1-33-3 \\
(312-314)\end{array}$} & & \\
\hline \multicolumn{2}{|r|}{$\begin{array}{r}\text { Discoaster } \\
\text { calcaris }\end{array}$} & $\begin{array}{c}\text { Within } \\
62.1-33-3 \\
(314)\end{array}$ & \multicolumn{2}{|l|}{$\begin{array}{r}\text { B Discoaster } \\
\quad \text { hamatus }\end{array}$} & & \\
\hline B & $\begin{array}{l}\text { Catinaster } \\
\text { calyculus }\end{array}$ & $\begin{array}{l}62.1-33-3 \\
62.1-33-C C \\
(314-319)\end{array}$ & $\begin{array}{c}\text { B Globigerinoides } \\
\text { adriaticus }\end{array}$ & $\begin{array}{l}62.1-33-3 \\
62.1-34-1 \\
(314-321)\end{array}$ & & \\
\hline
\end{tabular}


TABLE 1 - Continued

\begin{tabular}{|c|c|c|c|c|c|c|}
\hline \multicolumn{3}{|c|}{ Calcareous Nannofossil Events } & \multicolumn{2}{|c|}{ Foraminiferal Events } & \multicolumn{2}{|c|}{ Radiolarian Events } \\
\hline B & $\begin{array}{l}\text { Catinaster } \\
\quad \text { coalitus }\end{array}$ & $\begin{array}{l}62.1-33-3 \\
62.1-33-C C \\
(314-319)\end{array}$ & $\begin{array}{l}\text { B Globorotalia } \\
\text { merotumida }\end{array}$ & $\begin{array}{l}62.1-33-3 \\
62.1-34-1 \\
(314-321)\end{array}$ & $\begin{array}{l}\text { Cyclampterium tanythorax } \\
\text { C. brachythorax }\end{array}$ & $\begin{array}{l}62.1-33-2 \\
62.1-34-3 \\
(312-324) \\
\end{array}$ \\
\hline $\mathrm{T}$ & $\begin{array}{l}\text { Discoaster } \\
\text { exilis }\end{array}$ & $\begin{array}{l}62.1-33-C C \\
62.1-34-1 \\
(319-321) \\
\end{array}$ & & & $\begin{array}{c}\text { T Stichocorys } \\
\text { armata }\end{array}$ & $?$ \\
\hline \multirow[b]{5}{*}{ B } & \multirow[b]{5}{*}{$\begin{array}{l}\text { Discoaster } \\
\quad \text { challengeri }\end{array}$} & \multirow[b]{5}{*}{$\begin{array}{l}62.1-34-6 \\
62.1-34-\mathrm{CC} \\
(328)\end{array}$} & $\begin{array}{c}\mathrm{T} \text { Globorotalia } \\
\text { praemenardii } \\
\text { praemenardii }\end{array}$ & $\begin{array}{l}62.1-34-2 \\
62.1-34-3 \\
(322-324)\end{array}$ & $\begin{array}{l}\mathrm{T} \text { Dorcadospyris } \\
\text { alata }\end{array}$ & $\begin{array}{l}62.1-33-\mathrm{CC} \\
62.1-34-3 \\
(319-324) \\
\end{array}$ \\
\hline & & & $\begin{array}{c}\text { B Globorotalia } \\
\text { cultrata } \\
\text { menardii }\end{array}$ & $\begin{array}{l}62.1-34-4 \\
62.1-34-5 \\
(325-327) \\
\end{array}$ & & \\
\hline & & & $\begin{array}{c}\mathrm{T} \text { Globigerinita } \\
\text { parvula }\end{array}$ & $\begin{array}{l}62.1-34-5 \\
62.1-34-6 \\
(327-328)\end{array}$ & $\begin{array}{c}\mathrm{T} \text { Cyrtocapsella } \\
\text { cornuta }\end{array}$ & $\begin{array}{l}62.1-34-5 \\
62.1-34-C C \\
(327-328) \\
\end{array}$ \\
\hline & & & $\begin{array}{c}\text { T Globigerina } \\
\text { praebulloides } \\
\text { praebulloides }\end{array}$ & $\begin{array}{c}62.1-34-6 \\
62.1-35-1 \\
(328) \\
\end{array}$ & & \multirow{14}{*}{. } \\
\hline & & & $\begin{array}{c}\text { T Globigerinoides } \\
\text { subquadratus }\end{array}$ & $\begin{array}{c}62.1-34-6 \\
62.1-35-1 \\
(328)\end{array}$ & & \\
\hline \multirow[t]{4}{*}{$\mathrm{T}$} & \multirow[t]{4}{*}{$\begin{array}{l}\text { Discoaster } \\
\text { trinidadensis }\end{array}$} & \multirow[t]{4}{*}{$\begin{array}{c}62.1-34-\mathrm{CC} \\
62.1-35-1 \\
(328) \\
\end{array}$} & $\begin{array}{c}\text { B Globorotalia } \\
\text { cultrata } \\
\text { cultrata }\end{array}$ & $\begin{array}{c}62.1-34-6 \\
62.1-35-1 \\
(328)\end{array}$ & & \\
\hline & & & $\begin{array}{c}\mathrm{T} \text { Globorotalia } \\
\text { mayeri }\end{array}$ & $\begin{array}{c}62.1-34-6 \\
62.1-35-1 \\
(328) \\
\end{array}$ & & \\
\hline & & & $\begin{array}{c}\text { B Globigerina } \\
\text { nepenthes } \\
\text { picassiana }\end{array}$ & $\begin{array}{l}62.1-35-1 \\
62.1-35-2 \\
(328-329) \\
\end{array}$ & & \\
\hline & & & $\begin{array}{l}\text { T Globorotalia } \\
\text { aff. } \\
\text { peripheroacuta }\end{array}$ & $\begin{array}{l}62.1-35-1 \\
62.1-35-2 \\
(328-329)\end{array}$ & & \\
\hline \multirow[t]{4}{*}{ B } & \multirow[t]{4}{*}{$\begin{array}{l}\text { Discoaster } \\
\quad \text { extensus }\end{array}$} & $\begin{array}{l}62.1-35-2 \\
62.1-35-3 \\
(329-331)\end{array}$ & $\begin{array}{c}\text { B Globorotalia } \\
\text { cultrata } \\
\text { limbata }\end{array}$ & $\begin{array}{l}62.1-35-2 \\
62.1-35-3 \\
(329-331)\end{array}$ & & \\
\hline & & & $\begin{array}{c}\mathrm{T} \text { Globorotalia } \\
\text { minima }\end{array}$ & $\begin{array}{l}62.1-35-2 \\
62.1-35-3 \\
(329-331) \\
\end{array}$ & & \\
\hline & & & $\begin{array}{l}\mathrm{T} \text { Globigerina } \\
\text { nepenthoides }\end{array}$ & $\begin{array}{l}62.1-35-4 \\
62.1-35-5 \\
(332-334)\end{array}$ & & \\
\hline & & & $\begin{array}{c}\text { T Globorotalia } \\
\text { fohsi }\end{array}$ & $\begin{array}{l}62.1-35-4 \\
62.1-35-5 \\
(332-334)\end{array}$ & & \\
\hline \multirow[t]{2}{*}{$\overline{\mathrm{B}}$} & $\begin{array}{r}\text { Discoaster } \\
\text { kugleri }\end{array}$ & $\begin{array}{l}62.1-35-4 \\
62.1-35-5 \\
(332-334) \\
\end{array}$ & $\begin{array}{c}\text { T Globorotalia } \\
\quad \begin{array}{c}\text { praemenardii } \\
\text { archaeomenardii }\end{array}\end{array}$ & $\begin{array}{l}62.1-35-4 \\
62.1-35-5 \\
(332-334)\end{array}$ & & \\
\hline & & & $\begin{array}{c}\text { B } \begin{array}{c}\text { Hastigerinella } \\
\text { digitata } \\
\text { praedigitata }\end{array} \\
\end{array}$ & $\begin{array}{l}62.1-35-5 \\
62.1-35-6 \\
(334-335)\end{array}$ & & \\
\hline \multirow[t]{2}{*}{ B } & $\begin{array}{l}\text { Discoaster } \\
\quad \text { pseudovariabilis }\end{array}$ & $\begin{array}{l}62.1-35-5 \\
62.1-35-6 \\
(334-335)\end{array}$ & $\begin{array}{c}\text { B Globigerinita } \\
\text { glutinata } \\
\text { parkerae }\end{array}$ & $\begin{array}{l}62.1-35-5 \\
62.1-35-6 \\
(334-335)\end{array}$ & & \\
\hline & & & $\begin{array}{l}\text { T Hastigerina } \\
\text { siphonifera } \\
\text { praesiphonifera }\end{array}$ & $\begin{array}{l}62.1-35-6 \\
62.1-36-1 \\
(335-337)\end{array}$ & & \\
\hline
\end{tabular}


TABLE 1 - Continued

\begin{tabular}{|c|c|c|c|c|c|}
\hline \multicolumn{2}{|c|}{ Calcareous Nannofossil Events } & \multicolumn{2}{|c|}{ Foraminiferal Events } & \multicolumn{2}{|c|}{ Radiolarian Events } \\
\hline & & $\begin{array}{r}\text { B Hastigerina } \\
\text { siphonifera } \\
\text { siphonifera }\end{array}$ & $\begin{array}{l}62.1-35-6 \\
62.1-36-1 \\
(335-337)\end{array}$ & & \\
\hline & & $\begin{array}{r}\text { B Orbulina } \\
\text { universa } \\
\text { bilobata }\end{array}$ & $\begin{array}{l}62.1-35-6 \\
62.1-36-1 \\
(335-337)\end{array}$ & & \\
\hline & & $\begin{array}{r}\text { B Globigerina } \\
\text { bulbosa }\end{array}$ & $\begin{array}{l}62.1-36-3 \\
62.1-36-4 \\
(340-341)\end{array}$ & & \\
\hline & & $\begin{array}{l}\text { T Globorotalia } \\
\text { fohsi lobata }\end{array}$ & $\begin{array}{l}62.1-36-3 \\
62.1-36-4 \\
(340-341)\end{array}$ & & \\
\hline $\begin{array}{r}\text { D Discoaster } \\
\text { nephados }\end{array}$ & $\begin{array}{l}62.1-36-\mathrm{CC} \\
62.1-37-2 \\
(344-346) \\
\end{array}$ & $\begin{array}{l}\text { B Globorotalia } \\
\text { lenguaensis }\end{array}$ & $\begin{array}{l}62.1-36-5 \\
62.1-37-1 \\
(343-345) \\
\end{array}$ & & \\
\hline & & $\begin{array}{c}\text { B Globorotalia } \\
\text { praemenardii } \\
\text { praemenardii }\end{array}$ & $\begin{array}{l}62.1-37-2 \\
62.1-38-1 \\
(346-351) \\
\end{array}$ & $\begin{array}{c}\text { T Stichocorys } \\
\text { wolffii }\end{array}$ & $\begin{array}{l}62.1-37-2 \\
62.0-4-1 \\
(346-396) \\
\end{array}$ \\
\hline & & & & $\begin{array}{c}\text { B Artostrobium } \\
\text { doliolum }\end{array}$ & $\begin{array}{l}62.1-37-\mathrm{CC} \\
62.0-4-3 \\
(350-399)\end{array}$ \\
\hline & & & & $\begin{array}{l}\text { B Cannartus } \\
\text { petterssoni }\end{array}$ & $\begin{array}{l}62.1-35-2 \\
62.1-35-5 \\
(329-334) \\
\end{array}$ \\
\hline & & & & $\begin{array}{c}\text { T Carpocanopsis } \\
\text { bramlettei }\end{array}$ & $\begin{array}{l}62.1-35-5 \\
62.1-36-4 \\
(334-341)\end{array}$ \\
\hline & & & & $\begin{array}{c}\text { B Liriospyris } \\
\text { elevata }\end{array}$ & $\begin{array}{l}62.1-37-2 \\
62.1-37-\mathrm{CC} \\
(346-350)\end{array}$ \\
\hline & & & & $\begin{array}{l}\text { Lithopera renzae } \\
\rightarrow L \text {. neotera }\end{array}$ & $\begin{array}{l}62.1-37-2 \\
62.1-37-\mathrm{CC} \\
(346-350) \\
\end{array}$ \\
\hline \multirow[t]{5}{*}{ B $C$} & $\begin{array}{l}62.1-38-1 \\
62.1-38-2 \\
(351-352)\end{array}$ & & & & \\
\hline & & $\begin{array}{l}\mathrm{T} \text { Globorotalia } \\
\text { minutissima }\end{array}$ & $\begin{array}{l}62.1-38-4 \\
62.1-39-1 \\
(355-359)\end{array}$ & $\begin{array}{l}\text { Cannartus mammiferus } \\
\rightarrow \text { C. laticonus }\end{array}$ & $\begin{array}{l}62.1-38-C C \\
62.0-4-1 \\
(358-396)\end{array}$ \\
\hline & & $\begin{array}{c}\text { B } \begin{array}{c}\text { Globorotalia } \\
\text { riedeli }\end{array} \\
\end{array}$ & $\begin{array}{l}62.1-38-4 \\
62.1-39-1 \\
(355-359) \\
\end{array}$ & $\begin{array}{c}\text { T Cyrtocapsella } \\
\text { tetrapera }\end{array}$ & $\begin{array}{l}62.1-37-C C \\
62.0-4-C C \\
(350-404) \\
\end{array}$ \\
\hline & & & & $\begin{array}{l}\text { B Lithopera } \\
\text { thornburgi }\end{array}$ & $\begin{array}{l}62.1-36-C C \\
62.1-37-2 \\
(344-346)\end{array}$ \\
\hline & & & & $\begin{array}{r}\text { B Lithopera } \\
\text { baueri }\end{array}$ & $\begin{array}{l}62.1-35-2 \\
62.1-35-5 \\
(329-334) \\
\end{array}$ \\
\hline $\begin{array}{l}\text { B Triquetrorhabdulus } \\
\text { rugosus }\end{array}$ & $\begin{array}{l}62.1-39-1 \\
62.1-39-2 \\
(359-360) \\
\end{array}$ & & & & \\
\hline \multirow[t]{2}{*}{$\begin{array}{l}\mathrm{T} \text { Sphenolithus } \\
\quad \text { heteromorphus }\end{array}$} & $\begin{array}{l}62.1-39-3 \\
62.1-39-C C \\
(362-364) \\
\end{array}$ & & & & \\
\hline & & $\begin{array}{l}\text { T Cassigerinella } \\
\text { chipolensis }\end{array}$ & $\begin{array}{l}62.1-39-4 \\
64.1-1-1 \\
(363-434)\end{array}$ & & \\
\hline
\end{tabular}


TABLE 1 - Continued

\begin{tabular}{|c|c|c|c|c|}
\hline \multirow[t]{2}{*}{ Calcareous Nannofossil Events } & \multicolumn{2}{|c|}{ Foraminiferal Events } & \multicolumn{2}{|c|}{ Radiolarian Events } \\
\hline & $\begin{array}{r}\text { T Globigerina } \\
\text { multiloba }\end{array}$ & $\begin{array}{l}62.1-39-4 \\
64.1-1-1 \\
(363-434)\end{array}$ & $\begin{array}{r}\text { T Liriospyris } \\
\text { parkerae }\end{array}$ & $?$ \\
\hline & $\begin{array}{c}\mathrm{T} \text { Globigerina } \\
\text { praebulloides } \\
\text { leroyi }\end{array}$ & $\begin{array}{l}62.1-39-4 \\
64.1-1-1 \\
(363-434) \\
\end{array}$ & $\begin{array}{c}\text { B Siphocampe } \\
\text { corbula }\end{array}$ & $?$ \\
\hline & $\begin{array}{l}\text { T Globigerina } \\
\text { aff. quadrilatera }\end{array}$ & $\begin{array}{l}62.1-39-4 \\
64 \cdot 1-1-1 \\
(363-434)\end{array}$ & $\begin{array}{l}\text { Cyclampterium leptetrum } \\
\rightarrow \text { C. tanythorax }\end{array}$ & $\begin{array}{l}62.1-37-\mathrm{CC} \\
62.0-4-1 \\
(350-396)\end{array}$ \\
\hline & $\begin{array}{c}\mathrm{T} \text { Globigerinatella } \\
\text { insueta }\end{array}$ & $\begin{array}{l}62.1-39-4 \\
64.1-1-1 \\
(363-434) \\
\end{array}$ & $\begin{array}{c}\mathrm{T} \text { Calocycletta } \\
\text { virginis }\end{array}$ & $\begin{array}{l}62.1-37-\mathrm{CC} \\
62.0-4-1 \\
(350-396) \\
\end{array}$ \\
\hline & $\begin{array}{c}\text { T Globorotalia } \\
\text { obesa }\end{array}$ & $\begin{array}{l}62.1-39-4 \\
64.1-1-1 \\
(363-434)\end{array}$ & $\begin{array}{c}\text { T Psychospyris } \\
\text { grandis }\end{array}$ & $?$ \\
\hline & $\begin{array}{l}\mathrm{T} \text { Globorotalia } \\
\text { peripheroronda }\end{array}$ & $\begin{array}{l}62.1-39-4 \\
64.1-1-1 \\
(363-434)\end{array}$ & $\begin{array}{c}\mathrm{T} \text { Calocycletta } \\
\text { costata }\end{array}$ & $\begin{array}{l}62.1-37-\mathrm{CC} \\
62.0-4-1 \\
(350-396)\end{array}$ \\
\hline & $\begin{array}{r}\text { T Globorotalia } \\
\text { aff. riedeli }\end{array}$ & $\begin{array}{l}62.1-39-4 \\
64.1-1-1 \\
(363-434)\end{array}$ & $\begin{array}{c}\mathrm{T} \text { Dorcadospyris } \\
\text { dentata }\end{array}$ & $\begin{array}{l}62.1-37-\mathrm{CC} \\
62.0-4-1 \\
(350-396) \\
\end{array}$ \\
\hline & $\begin{array}{l}\mathrm{T} \text { Globigerina } \\
\text { ciperoensis } \\
\text { antustiumbilicata }\end{array}$ & $\begin{array}{l}64,1-1-1 \\
64.1-1-2 \\
(454-435)\end{array}$ & & \\
\hline & $\begin{array}{c}\text { T Globigerina } \\
\text { foliata }\end{array}$ & $\begin{array}{l}64.1-1-1 \\
64.1-1-2 \\
(434-435)\end{array}$ & & \\
\hline & T $\begin{array}{c}\text { Globigerinita } \\
\text { dissimilis }\end{array}$ & $\begin{array}{l}64.1-1-1 \\
64.1-1-2 \\
(434-435)\end{array}$ & & \\
\hline & $\begin{array}{r}\text { T Globigerinoides } \\
\text { altiaperturus }\end{array}$ & $\begin{array}{l}64.1-1-1 \\
64.1-1-2 \\
(434-435)\end{array}$ & & \\
\hline & $\begin{array}{c}\text { T Globigerinoides } \\
\text { quadrilobatus } \\
\text { praeimmaturus }\end{array}$ & $\begin{array}{l}64.1-1-1 \\
64.1-1-2 \\
(434-435)\end{array}$ & & \\
\hline & $\begin{array}{c}\mathrm{T} \text { Globigerinoides } \\
\text { sicanus }\end{array}$ & $\begin{array}{l}64.1-1-1 \\
64.1-1-2 \\
(434-435)\end{array}$ & & \\
\hline & $\begin{array}{r}\text { T Globorotalia } \\
\text { birnageae }\end{array}$ & $\begin{array}{l}64.1-1-1 \\
64.1-1-2 \\
(434-435)\end{array}$ & & \\
\hline & $\begin{array}{c}\text { T Globorotaloides } \\
\text { variabilis group }\end{array}$ & $\begin{array}{l}64.1-1-1 \\
64.1-1-2 \\
(434-435)\end{array}$ & & \\
\hline & $\begin{array}{c}\text { T Praeorbulina } \\
\text { glomerosa } \\
\text { curva }\end{array}$ & $\begin{array}{l}64.1-1-1 \\
64.1-1-2 \\
(434-435)\end{array}$ & & \\
\hline & $\begin{array}{r}\text { T Praeorbulina } \\
\text { transitoria }\end{array}$ & $\begin{array}{l}64.1-1-1 \\
64.1-1-2 \\
(434-435)\end{array}$ & $\begin{array}{c}\text { B Dorcadospyris } \\
\text { alata }\end{array}$ & $\begin{array}{l}64.1-1-3 \\
64.1-1-4 \\
(437-438)\end{array}$ \\
\hline & $\begin{array}{c}\text { B Praeorbulina } \\
\text { glomerosa } \\
\text { curva }\end{array}$ & $\begin{array}{l}64.1-1-2 \\
64.1-1-3 \\
(435-437)\end{array}$ & $\begin{array}{l}\text { Psychospyris intermedia } \\
\rightarrow P . \text { grandis }\end{array}$ & $?$ \\
\hline & $\begin{array}{l}\text { T Globigerina } \\
\text { pseudodruryi }\end{array}$ & $\begin{array}{l}64.1-1-4 \\
64.1-1-5 \\
(438-440)\end{array}$ & $\begin{array}{l}\text { Liriospyris stauropora } \\
\rightarrow \text { L. parkerae }\end{array}$ & $\begin{array}{l}64.1-1-4 \\
64.1-1-5 \\
(438-440)\end{array}$ \\
\hline
\end{tabular}


TABLE 1 - Continued

\begin{tabular}{|c|c|c|c|c|c|}
\hline \multicolumn{2}{|c|}{ Calcareous Nannofossil Events } & \multicolumn{2}{|c|}{ Foraminiferal Events } & \multicolumn{2}{|c|}{ Radiolarian Events } \\
\hline & & \multirow[t]{4}{*}{$\begin{array}{c}\text { T } \begin{array}{c}\text { Globigerinoides } \\
\text { subquadratus } \\
\text { subelongatus }\end{array} \\
\end{array}$} & \multirow[t]{4}{*}{$\begin{array}{l}64.1-1-4 \\
64.1-1-5 \\
(438-440)\end{array}$} & $\begin{array}{l}\text { Cannartus violina } \\
\rightarrow \text { C. mammiferus }\end{array}$ & \multirow{2}{*}{$\begin{array}{l}64.1-1-3 \\
64.1-1-5 \\
(437-440) \\
64.0-5-4 \\
64 \cdot 1-1-5 \\
(414-434)\end{array}$} \\
\hline & & & & $\begin{array}{r}\text { B Lithopera } \\
\text { renzae }\end{array}$ & \\
\hline & & & & $\begin{array}{c}\text { T Dorcadospyris } \\
\text { forcipata }\end{array}$ & $\begin{array}{l}64.1-1-3 \\
64.1-1-4 \\
(437-438)\end{array}$ \\
\hline & & & & $\begin{array}{c}\text { T Carpocanopsis } \\
\text { favosum }\end{array}$ & $\begin{array}{l}64.1-1-3 \\
64.1-1-5 \\
(437-440) \\
\end{array}$ \\
\hline & & $\begin{array}{c}\text { T Globigerinita } \\
\text { stainforthi } \\
\text { stainforthi }\end{array}$ & $\begin{array}{l}64.1-1-5 \\
64.1-1-6 \\
(440-441)\end{array}$ & & \\
\hline & & $\begin{array}{l}\text { B Praeorbulina } \\
\text { transitoria }\end{array}$ & $\begin{array}{l}64.1-1-5 \\
64.1-1-6 \\
(440-441)\end{array}$ & & \\
\hline & & $\begin{array}{l}\text { B Globorotalia } \\
\text { praemenardii } \\
\text { archaeomenardii }\end{array}$ & $\begin{array}{l}64.1-1-6 \\
64.1-2-1 \\
(441-443)\end{array}$ & & \\
\hline & & $\begin{array}{c}\text { T Globorotalia } \\
\text { scitula } \\
\text { praescitula }\end{array}$ & $\begin{array}{l}64.1-1-6 \\
64.1-2-1 \\
(441-443)\end{array}$ & & \\
\hline & & $\begin{array}{l}\text { T Globorotalia } \\
\text { peripheroronda } \\
\text { forma } \alpha\end{array}$ & $\begin{array}{l}64.1-2-2 \\
64.1-2-3 \\
(444-446)\end{array}$ & & \\
\hline \multirow[t]{10}{*}{$\begin{array}{c}\text { B Discoaster } \\
\text { exilis }\end{array}$} & \multirow[t]{10}{*}{$\begin{array}{l}64.1-2-6 \\
64.1-3-1 \\
(450-452)\end{array}$} & $\begin{array}{c}\text { T Globigerinita } \\
\text { unicava } \\
\text { unicava }\end{array}$ & $\begin{array}{l}64.1-2-6 \\
64.1-3-1 \\
(450-452)\end{array}$ & & \\
\hline & & $\begin{array}{c}\text { B Globigerinoides } \\
\text { sicanus }\end{array}$ & $\begin{array}{l}64.1-3-1 \\
64.1-3-2 \\
(452-453)\end{array}$ & & \\
\hline & & $\begin{array}{l}\text { B Globigerina } \\
\text { falconensis } \\
\text { falconensis }\end{array}$ & $\begin{array}{l}64.1-3-2 \\
64.1-3-3 \\
(453-455)\end{array}$ & $\begin{array}{l}\mathrm{T} \text { Cannartus } \\
\text { prismaticus }\end{array}$ & $\begin{array}{l}64.1-3-1 \\
64.1-3-3 \\
(452-455)\end{array}$ \\
\hline & & $\begin{array}{l}\text { B Globorotalia } \\
\text { continuosa }\end{array}$ & $\begin{array}{l}64.1-3-2 \\
64.1-3-3 \\
(453-455)\end{array}$ & & \\
\hline & & $\begin{array}{l}\text { B Globorotalia } \\
\text { scitula } \\
\text { praescitula }\end{array}$ & $\begin{array}{l}64 \cdot 1-3-2 \\
64 \cdot 1-3-3 \\
(453-455)\end{array}$ & & \\
\hline & & $\begin{array}{l}\text { B Hastigerina } \\
\quad \text { siphonifera } \\
\text { praesiphonifera }\end{array}$ & $\begin{array}{l}64.1-3-3 \\
64.1-3-4 \\
(455-456)\end{array}$ & & \\
\hline & & & & $\begin{array}{l}\text { T Stichocorys } \\
\text { diploconus }\end{array}$ & $\begin{array}{l}64.1-3-C C \\
64.1-4-1 \\
(460-462)\end{array}$ \\
\hline & & & & $\begin{array}{l}\text { B Phormostichoartus } \\
\text { corona }\end{array}$ & $\begin{array}{l}64.1-3-C C \\
64.1-4-1 \\
(460-462)\end{array}$ \\
\hline & & $\begin{array}{l}\text { B Globorotalia } \\
\text { minima }\end{array}$ & $\begin{array}{l}64.1-4-2 \\
64.1-4-3 \\
(463-465)\end{array}$ & & \\
\hline & & $\begin{array}{l}\text { B Globorotalia } \\
\text { scitula group }\end{array}$ & $\begin{array}{l}64.1-4-2 \\
64 \cdot 1-4-3 \\
(463-465)\end{array}$ & $\begin{array}{r}\text { T Carpocanopsis } \\
\text { cingulatum }\end{array}$ & $\begin{array}{l}64.1-3-3 \\
64.1-4-3 \\
(455-465)\end{array}$ \\
\hline
\end{tabular}


TABLE 1 - Continued

\begin{tabular}{|c|c|c|c|c|c|}
\hline \multicolumn{2}{|c|}{ Calcareous Nannofossil Events } & \multicolumn{2}{|c|}{ Foraminiferal Events } & \multicolumn{2}{|c|}{ Radiolarian Events } \\
\hline & & $\begin{array}{c}\text { B Globigerinoides } \\
\text { subquadratus } \\
\text { subelongatus }\end{array}$ & $\begin{array}{l}64.1-4-6 \\
64.1-5-1 \\
(469-471)\end{array}$ & $\begin{array}{c}\text { B Carpocanopsis } \\
\text { cristatum }\end{array}$ & $\begin{array}{l}64.1-4-3 \\
64.1-5-1 \\
(465-471)\end{array}$ \\
\hline & & $\begin{array}{c}\text { B Globigerinoides } \\
\text { bollii }\end{array}$ & $\begin{array}{l}64.1-5-2 \\
64.1-5-3 \\
(472-474)\end{array}$ & & \\
\hline & & $\begin{array}{r}\text { B Globigerina } \\
\text { hexagona }\end{array}$ & $\begin{array}{l}64.1-5-3 \\
64.1-5-4 \\
(474-475)\end{array}$ & & \\
\hline & & $\begin{array}{c}\text { B Globigerinoides } \\
\text { quadrilobatus } \\
\text { sacculifer typica }\end{array}$ & $\begin{array}{l}64.1-5-5 \\
64.1-5-6 \\
(477-478)\end{array}$ & & \\
\hline & & $\begin{array}{l}\text { B Globorotalia } \\
\text { peripheroronda }\end{array}$ & $\begin{array}{l}64.1-5-5 \\
64.1-5-6 \\
(477-478)\end{array}$ & & \\
\hline & & $\begin{array}{l}\text { B Globigerina } \\
\text { nepenthoides }\end{array}$ & $\begin{array}{l}64.1-5-6 \\
64.1-6-1 \\
(478-566)\end{array}$ & & \\
\hline & & $\begin{array}{l}\text { B Globigerina } \\
\text { pseudodruryi }\end{array}$ & $\begin{array}{l}64.1-5-6 \\
64.1-6-1 \\
(478-566)\end{array}$ & $\begin{array}{c}\text { B Calocycletta } \\
\text { costata }\end{array}$ & $\begin{array}{l}64.1-5-\mathrm{CC} \\
64.0-6-1 \\
(479-506)\end{array}$ \\
\hline & & $\begin{array}{c}\mathrm{T} \text { Globigerina } \\
\text { sellii }\end{array}$ & $\begin{array}{l}64.1-5-6 \\
64.1-6-1 \\
(478-566)\end{array}$ & & \\
\hline & & $\begin{array}{r}\text { T Globigerina } \\
\text { tripartita }\end{array}$ & $\begin{array}{l}64.1-5-6 \\
64.1-6-1 \\
(478-566)\end{array}$ & $\mathrm{T} \begin{array}{c}\text { Lychnocanium } \\
\text { bipes }\end{array}$ & $\begin{array}{l}64.1-5-1 \\
64.1-5-3 \\
(471-474) \\
\end{array}$ \\
\hline & & $\begin{array}{c}\text { B Globigerinatella } \\
\text { insueta }\end{array}$ & $\begin{array}{l}64.1-5-6 \\
64.1-6-1 \\
(478-566)\end{array}$ & & \\
\hline & & $\begin{array}{c}\text { B Globigerinoides } \\
\text { quadrilobatus } \\
\text { praeimmaturus }\end{array}$ & $\begin{array}{l}64.1-5-6 \\
64.1-6-1 \\
(478-566)\end{array}$ & $\begin{array}{r}\text { B Stichocorys } \\
\text { diploconus }\end{array}$ & $\begin{array}{l}64.1-4-C C \\
64.1-5-1 \\
(470-471)\end{array}$ \\
\hline \multirow[t]{2}{*}{$\begin{array}{r}\text { Sphenolithus } \\
\text { belemnos }\end{array}$} & $\begin{array}{l}64.1-5-1 \\
64.0-6-1 \\
(471-506)\end{array}$ & $\begin{array}{c}\text { T Globigerinoides } \\
\text { quadrilobatus } \\
\text { primordius }\end{array}$ & $\begin{array}{l}64.1-5-6 \\
64.1-6-1 \\
(478-566)\end{array}$ & & \\
\hline & & $\begin{array}{r}\text { B Globigerinoides } \\
\text { quadrilobatus } \\
\text { quadrilobatus }\end{array}$ & $\begin{array}{l}64.1-5-6 \\
64.1-6-1 \\
(478-566)\end{array}$ & $\begin{array}{c}\text { B Dorcadospyris } \\
\text { dentata }\end{array}$ & $\begin{array}{l}64.1-5-C C \\
64.0-6-1 \\
(479-506)\end{array}$ \\
\hline \multirow[t]{4}{*}{$\begin{array}{c}\mathrm{T} \text { Triquetrorhabdulus } \\
\text { carinatus }\end{array}$} & $\begin{array}{l}64.1-5-1 \\
64.0-6-1 \\
(471-506)\end{array}$ & $\begin{array}{c}\text { B Globigerinoides } \\
\text { quadrilobatus } \\
\text { trilobus }\end{array}$ & $\begin{array}{l}64.1-5-6 \\
64.1-6-1 \\
(478-566)\end{array}$ & & \\
\hline & & $\begin{array}{c}\text { B Globoquadrina } \\
\text { altispira } \\
\text { altispira }\end{array}$ & $\begin{array}{l}64.1-5-6 \\
64.1-6-1 \\
(478-566) \\
\end{array}$ & $\begin{array}{l}\text { Cannartus tubarius } \\
\rightarrow \text { C. violina }\end{array}$ & $\begin{array}{l}64.1-5-1 \\
64.1-5-C C \\
(471-479) \\
\end{array}$ \\
\hline & & $\begin{array}{c}\text { B Globoquadrina } \\
\text { altispira } \\
\text { globosa }\end{array}$ & $\begin{array}{l}64.1-5-6 \\
64.1-6-1 \\
(478-566)\end{array}$ & $\begin{array}{l}\text { T Theocorys } \\
\text { spongoconum }\end{array}$ & $\begin{array}{l}64.0-6-1 \\
64.0-6-4 \\
(506-510)\end{array}$ \\
\hline & & $\begin{array}{c}\text { B Globoquadrina } \\
\text { altispira } \\
\text { occlusa }\end{array}$ & $\begin{array}{l}64.1-5-6 \\
64.1-6-1 \\
(478-566)\end{array}$ & $\begin{array}{cc}\text { B } & \text { Liriospyris } \\
& \text { stauopora }\end{array}$ & $\begin{array}{l}\text { 64.1-5-CC } \\
64.0-6-1 \\
(479-506)\end{array}$ \\
\hline \multirow[t]{2}{*}{$\begin{array}{c}\text { T Discoaster } \\
\text { saundersi }\end{array}$} & $\begin{array}{l}64.0-6-5 \\
64.0-6-6 \\
(512-513) \\
\end{array}$ & $\begin{array}{r}\text { B Globorotalia } \\
\text { clemenciae }\end{array}$ & $\begin{array}{l}64.1-5-6 \\
64.1-6-1 \\
(478-566) \\
\end{array}$ & $\begin{array}{c}\text { T Botryopyle } \\
\text { sp. A }\end{array}$ & $\begin{array}{l}64.1-5-3 \\
64.0-6-1 \\
(474-506) \\
\end{array}$ \\
\hline & & $\begin{array}{c}\text { T Globorotalia } \\
\text { kugleri }\end{array}$ & $\begin{array}{l}64.1-5-6 \\
64.1-6-1 \\
(478-566)\end{array}$ & $\begin{array}{c}\text { B Stichocorys } \\
\text { wolffii }\end{array}$ & $\begin{array}{l}64.0-6-C C \\
64.1-6-1 \\
(514-566)\end{array}$ \\
\hline
\end{tabular}


TABLE 1 - Continued

\begin{tabular}{|c|c|c|c|c|c|}
\hline \multicolumn{2}{|c|}{ Calcareous Nannofossil Events } & \multicolumn{2}{|c|}{ Foraminiferal Events } & \multicolumn{2}{|c|}{ Radiolarian Events } \\
\hline & & $\begin{array}{r}\mathrm{T} \text { Globorotalia } \\
\text { mendacis }\end{array}$ & $\begin{array}{l}64.1-5-6 \\
64.1-6-1 \\
(478-566)\end{array}$ & $\begin{array}{l}\text { Cyclampterium pegetrum } \\
\rightarrow C \text {. leptetrum }\end{array}$ & $\begin{array}{l}64.0-6-\mathrm{CC} \\
64.1-6-1 \\
(514-566)\end{array}$ \\
\hline & & $\begin{array}{l}\text { B Globorotalia } \\
\text { peripheroronda } \\
\text { forma } \alpha\end{array}$ & $\begin{array}{l}64.1-5-6 \\
64.1-6-1 \\
(478-566)\end{array}$ & $\begin{array}{c}\text { B Carpocanopsis } \\
\text { bramlettei }\end{array}$ & $\begin{array}{l}64.1-6-3 \\
64.0-7-1 \\
(569-611)\end{array}$ \\
\hline \multirow[t]{9}{*}{$\begin{array}{r}\text { Biscoaster } \\
\text { aulakos }\end{array}$} & $\begin{array}{l}64.0-6-6 \\
64.1-6-1 \\
(513-566)\end{array}$ & $\begin{array}{r}\mathrm{T} \text { Globorotalia } \\
\text { aff. siakensis }\end{array}$ & $\begin{array}{l}64.1-5-6 \\
64.1-6-1 \\
(478-566)\end{array}$ & $\begin{array}{r}\text { B Cannartus } \\
\text { tubarius }\end{array}$ & $\begin{array}{l}64.0-6-\mathrm{CC} \\
64.1-6-1 \\
(514-566)\end{array}$ \\
\hline & & $\begin{array}{l}\text { B Globorotaloides } \\
\text { variabilis group }\end{array}$ & $\begin{array}{l}64.1-5-6 \\
64.1-6-1 \\
(478-566)\end{array}$ & $\begin{array}{c}\text { B Stichocorys } \\
\text { armata }\end{array}$ & $\begin{array}{l}64.0-6-1 \\
64.1-6-1 \\
(506-566)\end{array}$ \\
\hline & & $\begin{array}{c}\text { B Globigerina } \\
\text { foliata }\end{array}$ & $\begin{array}{l}64.1-6-1 \\
64.1-6-2 \\
(566-567)\end{array}$ & & \\
\hline & & $\begin{array}{l}\text { B Globigerina } \\
\text { aff. quadrilatera }\end{array}$ & $\begin{array}{l}64.1-6-2 \\
64.1-6-3 \\
(567-569)\end{array}$ & & \\
\hline & & $\begin{array}{c}\text { B Globigerinoides } \\
\text { sicanus } \\
\text { praesicanus }\end{array}$ & $\begin{array}{l}64.1-6-2 \\
64.1-6-3 \\
(567-569)\end{array}$ & & \\
\hline & & $\begin{array}{r}\text { B Globorotalia } \\
\text { birnageae }\end{array}$ & $\begin{array}{l}64.1-5-2 \\
64.1-6-3 \\
(567-569)\end{array}$ & & \\
\hline & & $\begin{array}{c}\text { T Globigerina } \\
\text { ciperoensis } \\
\text { angulisuturalis }\end{array}$ & $\begin{array}{l}64.1-6-3 \\
64 \cdot 1-6-4 \\
(569-570)\end{array}$ & & \\
\hline & & $\begin{array}{c}\text { B Globigerinoides } \\
\text { quadrilobatus } \\
\text { immaturus }\end{array}$ & $\begin{array}{l}64.1-6-3 \\
64.1-6-4 \\
(569-570)\end{array}$ & & \\
\hline & & 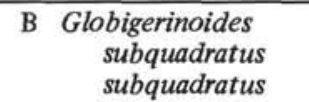 & $\begin{array}{l}64.1-6-3 \\
64.1-6-4 \\
(569-570)\end{array}$ & & \\
\hline \multirow[t]{2}{*}{$\begin{array}{c}\text { B Shenolithus } \\
\text { heteromorphus }\end{array}$} & $\begin{array}{l}64.1-6-4 \\
64.0-7-1 \\
(570-611)\end{array}$ & $\begin{array}{c}\text { B Globigerina } \\
\text { conglomerata } \\
\text { venezuelana }\end{array}$ & $\begin{array}{l}64.1-6-4 \\
64.1-7-2 \\
(570-663)\end{array}$ & $\begin{array}{c}\mathrm{T} \text { Theocyrtis } \\
\text { annosa }\end{array}$ & $\begin{array}{l}64.1-6-3 \\
64.0-7-1 \\
(569-611)\end{array}$ \\
\hline & & $\begin{array}{c}\text { B Globigerina } \\
\text { praebulloides } \\
\text { leroyi }\end{array}$ & $\begin{array}{l}64.1-6-4 \\
64.1-7-2 \\
(570-663)\end{array}$ & & \\
\hline B Discoaster & $64.0-7-3$ & $\begin{array}{r}\text { B Globigerina } \\
\text { praebulloides } \\
\text { praebulloides }\end{array}$ & $\begin{array}{l}64.1-6-4 \\
64.1-7-2 \\
(570-663)\end{array}$ & $\begin{array}{r}\text { B Stichocorys } \\
\text { delmontensis }\end{array}$ & $\begin{array}{l}64.1-6-C C \\
64.0-7-1 \\
(574-611)\end{array}$ \\
\hline \multirow[t]{5}{*}{$\begin{aligned} \text { B Discoaster } \\
\text { trinidadensis }\end{aligned}$} & $\begin{array}{l}64.0-7-3 \\
64.0-7-4 \\
(614-615)\end{array}$ & $\begin{array}{r}\text { T Globigerina } \\
\text { prasaepis }\end{array}$ & $\begin{array}{l}64.1-6-4 \\
64.1-7-2 \\
(570-663)\end{array}$ & $\begin{array}{c}\mathrm{T} \text { Dorcadospyris } \\
\text { ateuchus }\end{array}$ & $\begin{array}{l}\text { 64.0-7-CC } \\
64.1-7-1 \\
(619-662)\end{array}$ \\
\hline & & $\begin{array}{r}\mathrm{T} \text { Globigerinita } \\
\text { pera group }\end{array}$ & $\begin{array}{l}64.1-6-4 \\
64.1-7-2 \\
(570-663)\end{array}$ & $\begin{array}{c}\text { B Carpocanopsis } \\
\text { favosum }\end{array}$ & $\begin{array}{l}64.0-7-3 \\
64.1-7-3 \\
(614-665)\end{array}$ \\
\hline & & $\begin{array}{r}\text { B Globigerinita } \\
\text { stainforthi } \\
\text { stainforthi }\end{array}$ & $\begin{array}{l}64.1-6-4 \\
64.1-7-2 \\
(570-663) \\
\end{array}$ & $\begin{array}{c}\text { B Botryocyrtis } \\
\text { spp. }\end{array}$ & $\begin{array}{l}64.1-6-1 \\
64.1-6-3 \\
(566-569)\end{array}$ \\
\hline & & $\begin{array}{r}\text { B } \begin{array}{r}\text { Globigerinoides } \\
\text { altiaperturus }\end{array} \\
\end{array}$ & $\begin{array}{l}64.1-6-4 \\
64.1-7-2 \\
(570-663)\end{array}$ & $\begin{array}{c}\mathrm{T} \text { Dorcadospyris } \\
\text { papilio }\end{array}$ & $\begin{array}{l}64.0-7-\mathrm{CC} \\
64.1-7-1 \\
(619-662) \\
\end{array}$ \\
\hline & & $\begin{array}{c}\text { B Globigerinoides } \\
\text { obliquus } \\
\text { obliquus }\end{array}$ & $\begin{array}{l}64.1-6-4 \\
64.1-7-2 \\
(570-663)\end{array}$ & $\begin{array}{c}\text { B Cyrtocapsella } \\
\text { cornuta }\end{array}$ & $\begin{array}{l}64.0-7-\mathrm{CC} \\
64.1-7-1 \\
(619-662)\end{array}$ \\
\hline
\end{tabular}


TABLE 1 - Continued

\begin{tabular}{|c|c|c|c|c|c|c|}
\hline \multicolumn{3}{|c|}{ Calcareous Nannofossil Events } & \multicolumn{2}{|c|}{ Foraminiferal Events } & \multicolumn{2}{|c|}{ Radilarian Events } \\
\hline & & & $\begin{array}{c}\text { B Globigerinoides } \\
\text { quadrilobatus } \\
\text { primordius }\end{array}$ & $\begin{array}{l}64.1-6-4 \\
64.1-7-2 \\
(570-663)\end{array}$ & $\begin{array}{c}\text { B Calocycletta } \\
\text { virginis }\end{array}$ & $\begin{array}{l}64.0-7-\mathrm{CC} \\
64.1-7-1 \\
(619-662)\end{array}$ \\
\hline & & & $\begin{array}{l}\text { B Globoquadrina } \\
\text { dehiscens group }\end{array}$ & $\begin{array}{l}64.1-6-4 \\
64.1-7-2 \\
(570-663)\end{array}$ & $\begin{array}{l}\text { Psychospyris parva } \\
\rightarrow \text { P. intermedia }\end{array}$ & $?$ \\
\hline & & & $\begin{array}{c}\text { B Globorotalia } \\
\text { obesa }\end{array}$ & $\begin{array}{l}64.1-6-4 \\
64.1-7-2 \\
(570-663) \\
\end{array}$ & $\begin{array}{c}\text { B Cyrtocapsella } \\
\text { tetrapera }\end{array}$ & $\begin{array}{l}64.0-7-\mathrm{CC} \\
64.1-7-1 \\
(619-662)\end{array}$ \\
\hline & & & $\begin{array}{l}\mathrm{T} \text { Globorotalia } \\
\text { pseudokugleri }\end{array}$ & $\begin{array}{l}64.1-6-4 \\
64.1-7-2 \\
(570-663)\end{array}$ & & \\
\hline & & & $\begin{array}{r}\text { B } \begin{array}{c}\text { Globigerina } \\
\text { tripartita }\end{array} \\
\end{array}$ & $\begin{array}{l}64.1-7-2 \\
64.1-7-3 \\
(663-665)\end{array}$ & & \\
\hline & & & $\begin{array}{c}\text { B Globigerinita } \\
\text { glutinata } \\
\text { glutinata }\end{array}$ & $\begin{array}{l}64.1-7-2 \\
64.1-7-3 \\
(663-665)\end{array}$ & & \\
\hline & & & $\begin{array}{cc}\text { B } & \begin{array}{c}\text { Globigerinita } \\
\text { glutinata } \\
\text { juvenilis }\end{array} \\
\end{array}$ & $\begin{array}{l}64.1-7-2 \\
64.1-7-3 \\
(663-665)\end{array}$ & & \\
\hline & & & $\begin{array}{c}\mathrm{T} \text { Globorotalia } \\
\text { gemma }\end{array}$ & $\begin{array}{l}64.1-7-2 \\
64.1-7-3 \\
(663-665)\end{array}$ & & \\
\hline & & & $\begin{array}{c}\text { B Globorotalia } \\
\text { kugleri }\end{array}$ & $\begin{array}{l}64.1-7-2 \\
64.1-7-3 \\
(663-665)\end{array}$ & & \\
\hline & & & $\begin{array}{r}\text { B Globorotalia } \\
\text { mendacis }\end{array}$ & $\begin{array}{l}64.1-7-2 \\
64.1-7-3 \\
(663-665)\end{array}$ & & \\
\hline & & & $\begin{array}{l}\text { B Globorotalia } \\
\text { aff. riedel }\end{array}$ & $\begin{array}{l}64.1-7-2 \\
64 \cdot 1-7-3 \\
(663-665)\end{array}$ & & \\
\hline & & & $\begin{array}{c}\text { B Globigerina } \\
\text { sellii }\end{array}$ & $\begin{array}{l}64.1-7-3 \\
64.1-7-4 \\
(665-666)\end{array}$ & & \\
\hline & & & $\begin{array}{l}\text { B Globorotalia } \\
\text { minutissima }\end{array}$ & $\begin{array}{l}64.1-7-3 \\
64.1-7-4 \\
(665-666)\end{array}$ & $\begin{array}{c}\text { B Carpocanopsis } \\
\text { cingulatum }\end{array}$ & $\begin{array}{l}64.1-7-3 \\
64.0-8-2 \\
(665-707)\end{array}$ \\
\hline & & & $\begin{array}{c}\text { T Globorotalia } \\
\text { opima } \\
\text { nana }\end{array}$ & $\begin{array}{l}64.1-7-3 \\
64.1-7-4 \\
(665-666)\end{array}$ & $\begin{array}{c}\text { T Artophormis } \\
\text { gracilis }\end{array}$ & $\begin{array}{l}64.1-7-1 \\
64.1-8-1 \\
(662-747)\end{array}$ \\
\hline & & & $\begin{array}{c}\text { T Globorotalia } \\
\text { opima } \\
\text { opima }\end{array}$ & $\begin{array}{l}64.1-7-3 \\
64.1-7-4 \\
(665-666)\end{array}$ & $\begin{array}{c}\text { B Psychospyris } \\
\text { parva }\end{array}$ & $?$ \\
\hline & & & $\begin{array}{l}\mathrm{T} \text { Globigerina } \\
\quad \text { pseudovenezuelana }\end{array}$ & $\begin{array}{l}64.1-7-4 \\
64.1-8-1 \\
(667-747) \\
\end{array}$ & $\begin{array}{c}\text { T Centrobotrys } \\
\text { sp. A }\end{array}$ & $\begin{array}{l}64.0-7-1 \\
64.0-7-3 \\
(611-614) \\
\end{array}$ \\
\hline$T$ & $\begin{array}{c}\text { Sphenolithus } \\
\text { distentus }\end{array}$ & $\begin{array}{l}64.1-7-4 \\
64.0-8-1 \\
(666-706) \\
\end{array}$ & $\begin{array}{c}\text { B Globigerinita } \\
\text { uvula }\end{array}$ & $\begin{array}{l}64.1-7-4 \\
64.1-8-1 \\
(666-747) \\
\end{array}$ & $\begin{array}{c}\text { T Lychnocanium } \\
\text { trifolium }\end{array}$ & $\begin{array}{l}64.1-7-C C \\
64.0-8-1 \\
(670-706) \\
\end{array}$ \\
\hline B & $\begin{array}{r}\text { Sphenolithus } \\
\text { belemnos }\end{array}$ & $\begin{array}{l}64.1-7-4 \\
64.0-8-1 \\
(666-706)\end{array}$ & $\begin{array}{c}\text { T Globigerinita } \\
\text { unicava } \\
\text { primitiva }\end{array}$ & $\begin{array}{l}64.1-7-4 \\
64.1-8-1 \\
(666-747) \\
\end{array}$ & $\begin{array}{c}\mathrm{T} \text { Lithocyclia } \\
\text { angustum }\end{array}$ & $\begin{array}{l}64.1-7-C C \\
64.0-8-1 \\
(670-706)\end{array}$ \\
\hline $\mathrm{T}$ & $\begin{array}{c}\text { Sphenolithus } \\
\text { predistentus }\end{array}$ & $\begin{array}{l}64.0-8-1 \\
64.0-8-2 \\
(706-707)\end{array}$ & $\begin{array}{l}\text { B Globorotalia } \\
\text { pseudokugleri }\end{array}$ & $\begin{array}{l}64.1-7-4 \\
64.1-8-1 \\
(666-747)\end{array}$ & $\begin{array}{c}\text { B Lychnocanium } \\
\text { bipes }\end{array}$ & $\begin{array}{l}64.1-7-\mathrm{CC} \\
64.0-8-1 \\
(670-706)\end{array}$ \\
\hline
\end{tabular}


TABLE 1 - Continued

\begin{tabular}{|c|c|c|c|c|c|c|}
\hline \multicolumn{3}{|c|}{ Calcareous Nannoplankton Events } & \multicolumn{2}{|c|}{ Foraminiferal Events } & \multicolumn{2}{|c|}{ Radiolarian Events } \\
\hline $\mathrm{T}$ & $\begin{array}{l}\text { Coccolithus } \\
\text { eopelagicus }\end{array}$ & $\begin{array}{l}64.0-8-3 \\
64.1-8-1 \\
(709-747) \\
\end{array}$ & $\begin{array}{l}\text { B Turborotalita } \\
\text { humilis group }\end{array}$ & $\begin{array}{l}64.1-7-4 \\
64.1-8-1 \\
(666-747)\end{array}$ & $\begin{array}{c}\text { B Lychnocanium } \\
\text { trifolium }\end{array}$ & $\begin{array}{l}64.0-8-C C \\
64.1-8-1 \\
(714-747)\end{array}$ \\
\hline $\bar{T}$ & $\begin{array}{l}\text { Discoaster } \\
\quad \text { lidzi }\end{array}$ & $\begin{array}{l}64.0-8-3 \\
64.1-8-1 \\
(709-747)\end{array}$ & & & $\begin{array}{c}\text { B Botryopyle } \\
\text { sp. A }\end{array}$ & $\begin{array}{l}64.0-8-2 \\
64.1-8-1 \\
(707-747)\end{array}$ \\
\hline $\bar{B}$ & $\begin{array}{l}\text { Discoaster } \\
\text { nephados }\end{array}$ & $\begin{array}{l}64.0-8-3 \\
64.1-8-1 \\
(709-747) \\
\end{array}$ & & & $\begin{array}{l}\text { Cyclampterium milowi } \\
\rightarrow C \text {. pegetrum }\end{array}$ & $\begin{array}{l}64.1-8-1 \\
64.1-8-2\end{array}$ \\
\hline B & $\begin{array}{l}\text { Triquetrorhabdulus } \\
\text { carinatus }\end{array}$ & $\begin{array}{l}64.0-8-2 \\
64.1-8-1 \\
(709-747)\end{array}$ & . & & $\begin{array}{c}\text { B Dorcadospyris } \\
\text { papilio }\end{array}$ & $\begin{array}{l}64.0-8-\mathrm{CC} \\
64.1-8-1 \\
(714-747)\end{array}$ \\
\hline
\end{tabular}


TABLE 2

Relationship of Neogene Microfossil Zonations Observed at Sites 62 through 64

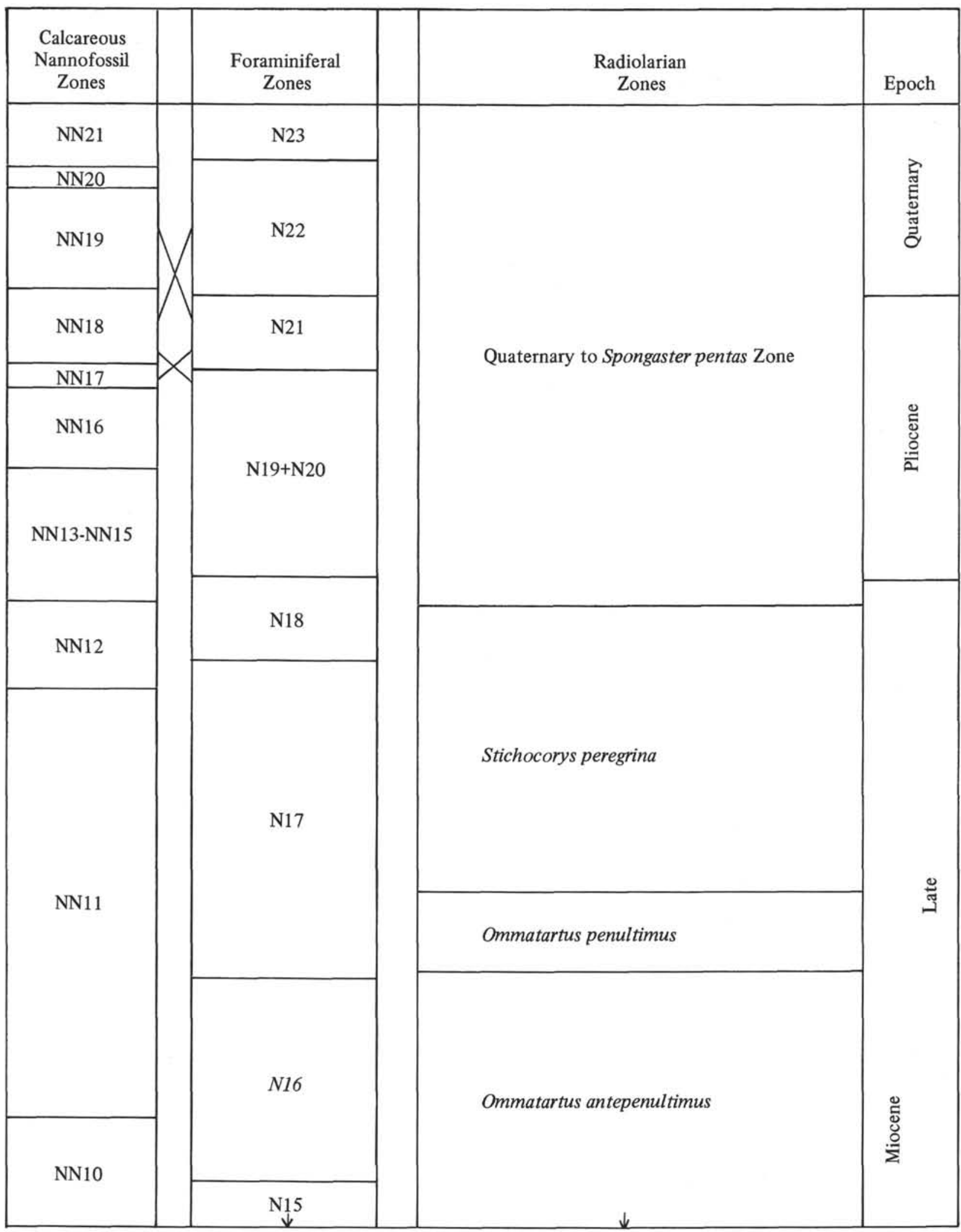


TABLE 2-Continued

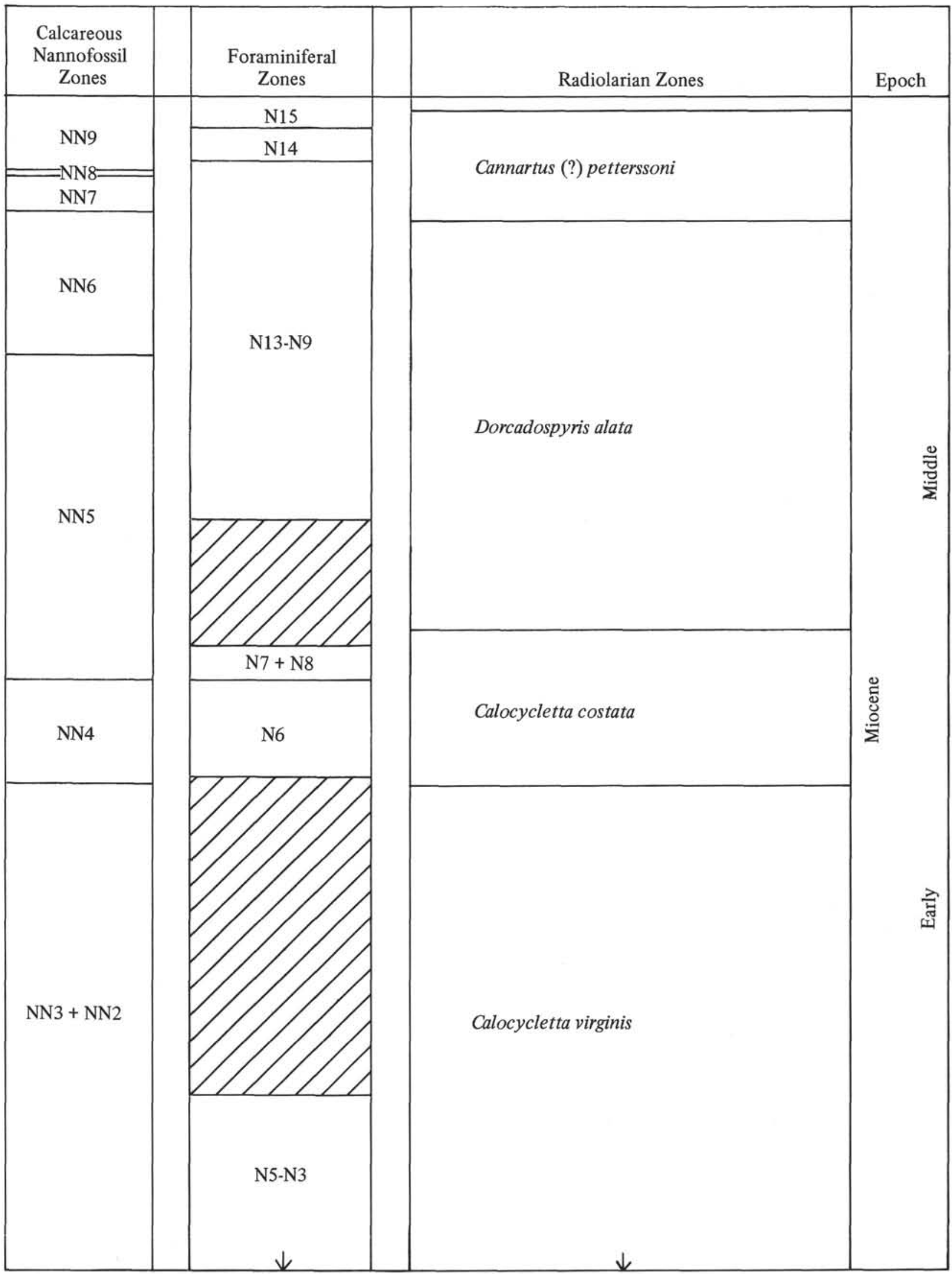


TABLE 2 - Continued

\begin{tabular}{|c|c|c|c|}
\hline $\begin{array}{c}\text { Calcareous } \\
\text { Nannofossil } \\
\text { Zones }\end{array}$ & $\begin{array}{c}\text { Foraminiferal } \\
\text { Zones }\end{array}$ & Radiolarian Zones & Epoch \\
\hline \multirow{3}{*}{ NN1 } & \multirow{2}{*}{ N5-N3 } & Calocycletta virginis & \multirow{3}{*}{ 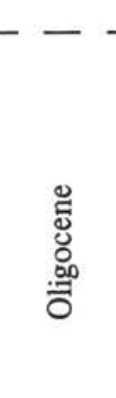 } \\
\hline & & \multirow[b]{2}{*}{ Lychnocanium bipes } & \\
\hline & & & \\
\hline
\end{tabular}




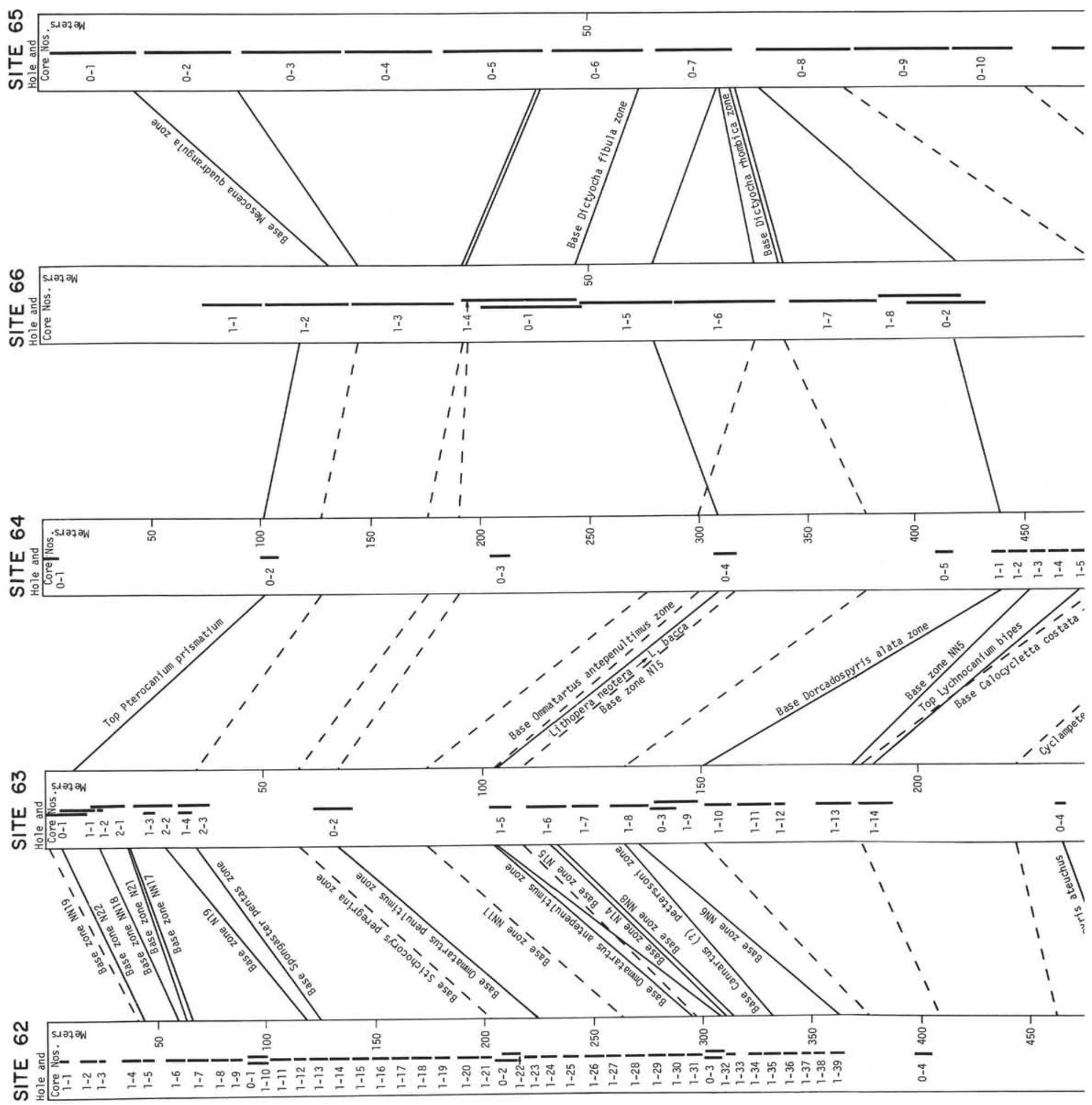



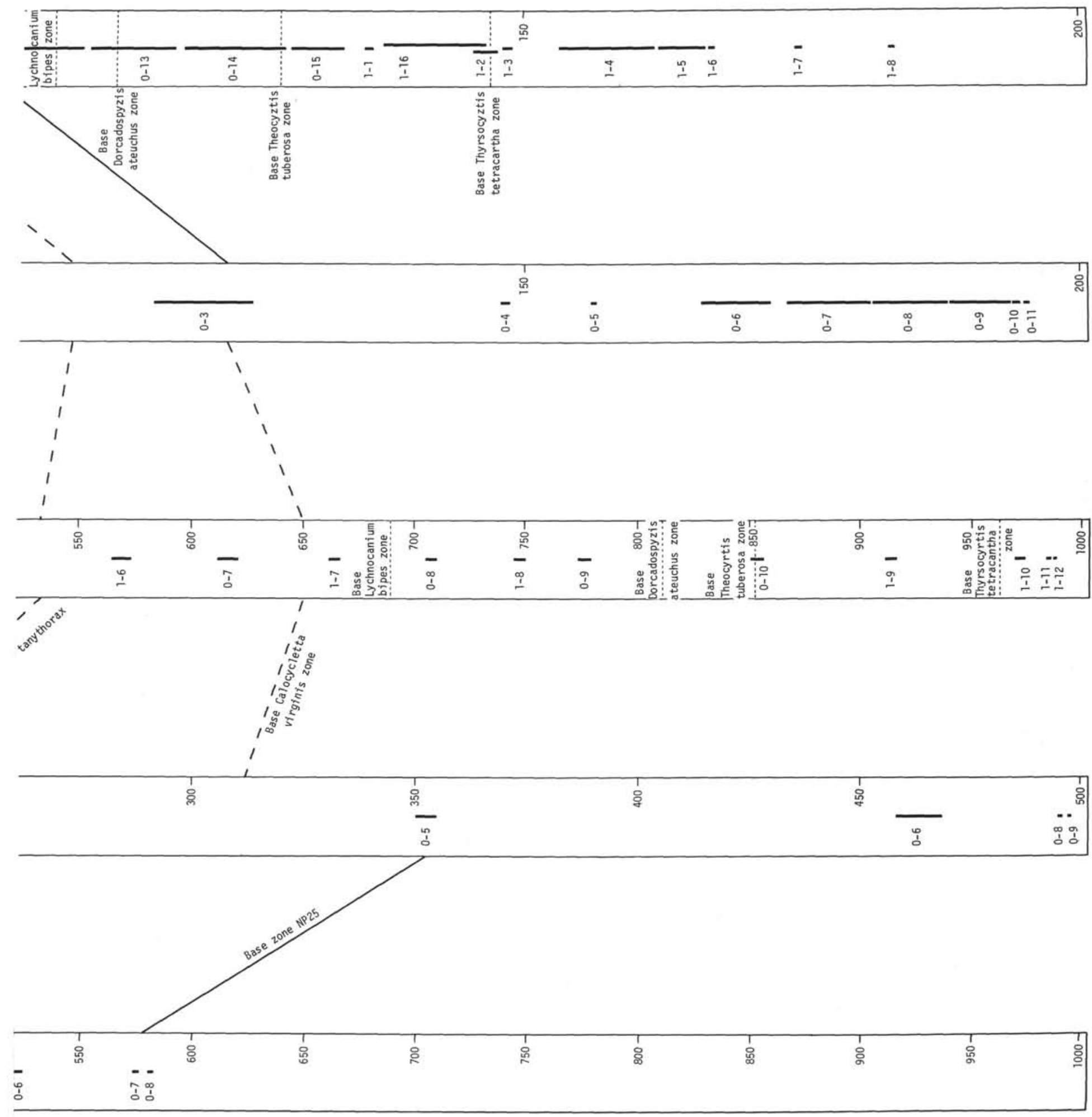

Figure 1. Simplified correlation-diagram of the sequences at Sites 62-66. 